\title{
Development of Glutamatergic Synaptic Transmission in Binaural
} Auditory Neurons

\author{
Jason Tait Sanchez, ${ }^{1,2}$ Yuan Wang, ${ }^{1,2}$ Edwin W Rubel, ${ }^{1-3}$ and Andres Barria ${ }^{1,3}$ \\ ${ }^{1}$ Virginia Merrill Bloedel Hearing Research Center and ${ }^{2}$ Departments of Otolaryngology_Head and Neck Surgery and ${ }^{3}$ Physiology and \\ Biophysics, University of Washington, Seattle, Washington
}

Submitted 26 May 2010; accepted in final form 23 July 2010

Tait Sanchez J, Wang Y, Rubel EW, Barria A. Development of glutamatergic synaptic transmission in binaural auditory neurons. $J$ Neurophysiol 104: 1774-1789, 2010. First published July 28, 2010; doi:10.1152/jn.00468.2010. Glutamatergic synaptic transmission is essential for binaural auditory processing in birds and mammals. Using whole cell voltage clamp recordings, we characterized the development of synaptic ionotropic glutamate receptor (iGluR) function from auditory neurons in the chick nucleus laminaris (NL), the first nucleus responsible for binaural processing. We show that synaptic transmission is mediated by AMPA- and $N$-methyl-D-aspartate (NMDA)-type glutamate receptors (AMPA-R and NMDA-R, respectively) when hearing is first emerging and dendritic morphology is being established across different sound frequency regions. Puff application of glutamate agonists at embryonic day 9 (E9) revealed that both iGluRs are functionally present prior to synapse formation (E10). Between E11 and E19, the amplitude of isolated AMPA-R currents from high-frequency (HF) neurons increased 14-fold. A significant increase in the frequency of spontaneous events is also observed. Additionally, AMPA-R currents become faster and more rectifying, suggesting developmental changes in subunit composition. These developmental changes were similar in all tonotopic regions examined. However, mid- and low-frequency neurons exhibit fewer spontaneous events and evoked AMPA-R currents are smaller, slower, and less rectifying than currents from age-matched HF neurons. The amplitude of isolated NMDA-R currents from HF neurons also increased, reaching a peak at E17 and declining sharply by E19, a trend consistent across tonotopic regions. With age, NMDA-R kinetics become significantly faster, indicating a developmental switch in receptor subunit composition. Dramatic increases in the amplitude and speed of glutamatergic synaptic transmission occurs in NL during embryonic development. These changes are first seen in HF neurons suggesting regulation by peripheral inputs and may be necessary to enhance coincidence detection of binaural auditory information.

\section{N T R O D U C T I O N}

In many brain regions, excitatory responses mediated by postsynaptic ionotropic glutamate receptors (iGluRs) are required for correct circuit formation and maturation (Espinosa et al. 2009; Hall and Ghosh 2008). During synaptic development, iGluRs often show remarkable modifications in their biophysical properties (Carmignoto and Vicini 1992; Esteban et al. 2003; Kumar et al. 2002; Quinlan et al. 1999; Tovar and Westbrook 1999), which coincide with changes in synaptic growth and pruning (Cline and Haas 2008) as well as alterations in dendritic structure and circuit refinement (Lichtman and Colman 2000). Typically, the AMPA-type glutamate receptor (AMPA-R) is necessary for rapid synaptic transmission,

\footnotetext{
Address for reprint requests and other correspondence: A. Barria, Physiology and Biophysics, School of Medicine, University of Washington, Box 357290, Seattle, WA 98195 (E-mail: barria@u.washington.edu).
}

whereas the $N$-methyl-D-aspartate (NMDA)-type glutamate receptor (NMDA-R) mediates a slower excitatory response thought to be critical for modulating synaptic function (Dingledine et al. 1999). Interestingly, little is known about the developmental role of iGluRs in the avian binaural auditory system, a relatively simple circuit extensively studied with respect to the development of structure and function.

In birds, nucleus laminaris (NL) is a third-order auditory brain stem structure responsible for binaural sound processing. The cell body layer of NL neurons are arranged according to the optimal frequency response of the neuron (i.e., tonotopic organization) and contain segregated dorsal and ventral aspiny dendrites that exhibit a systematic gradient in dendritic length (Fig. 1A) that runs parallel to this tonotopic axis (Parks and Rubel 1978; Smith and Rubel 1979). Functionally, mature NL neurons are highly specialized for encoding temporal information of sound (Kuba et al. 2006). These neurons possess a variety of physiological specializations (Kuba et al. 2005) that facilitate coincidence detection (Kuba et al. 2002a,b, 2003) thought essential for binaural hearing (Hyson 2005; Young and Rubel 1983).

Soon after synapses form [ embryonic day 10 (E10)], excitatory postsynaptic potentials (EPSPs) from NL neurons become extremely fast (Gao and Lu 2008) to enhance the ability of NL neurons to act as binaural coincident detectors (Raman et al. 1994; Reyes et al. 1996; Trussell 1997, 1999). This sharpening of the EPSP soon after synaptogenesis has been interpreted as a loss in the NMDA-R mediated component (Gao and Lu 2008), consistent with previous reports of the absence of NMDA-R responses later in development ( $>$ E16) (Funabiki et al. 1998; Zhou and Parks 1991). However, during this embryonic period of rich dendritic elaboration and circuit refinement, NMDA-Rs have clearly been shown, in NL (Tang and Carr 2004). Moreover, different NMDA-R subunits have specific temporal patterns of expression (Tang and Carr 2007), suggesting the presence of NMDA-Rs with different biophysical properties during the developmental period when the tonotopic gradient is being established.

In this report, we characterize the developmental profile and functional expression of iGluRs in NL neurons. We report dramatic changes in both AMPA-R and NMDA-R responses occurring differentially across the tonotopic gradient during a period when synapses are forming, specializations and circuitry are established, and hearing is emerging.

\section{METHODS}

\section{Slice preparation}

Acute brain stem slices were prepared from White Leghorn chicken (Gallus domesticus) embryos at E9, E10, E11, E13, E15, E17, and E19 
A
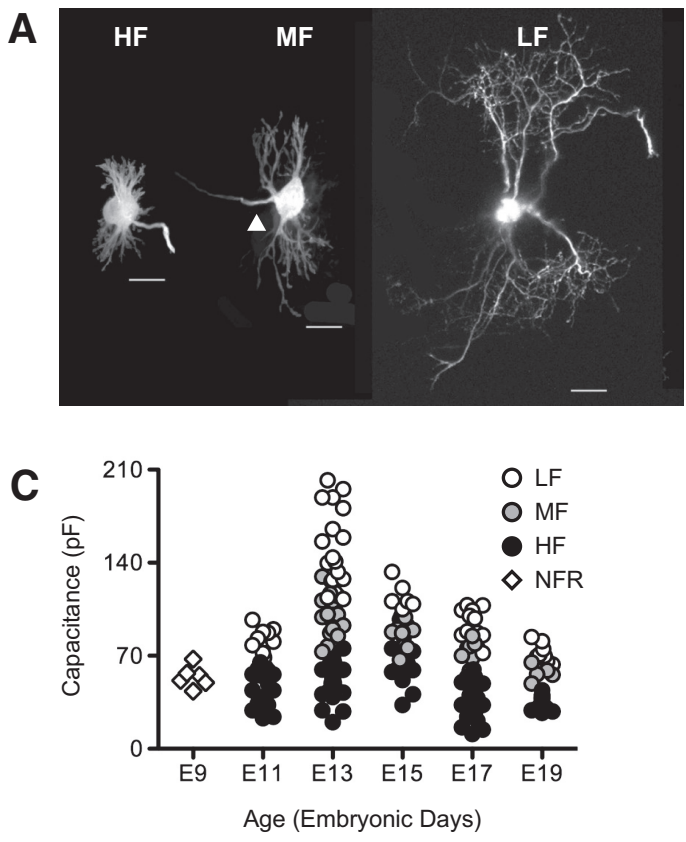

D

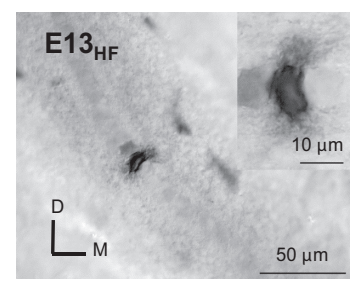

E
B
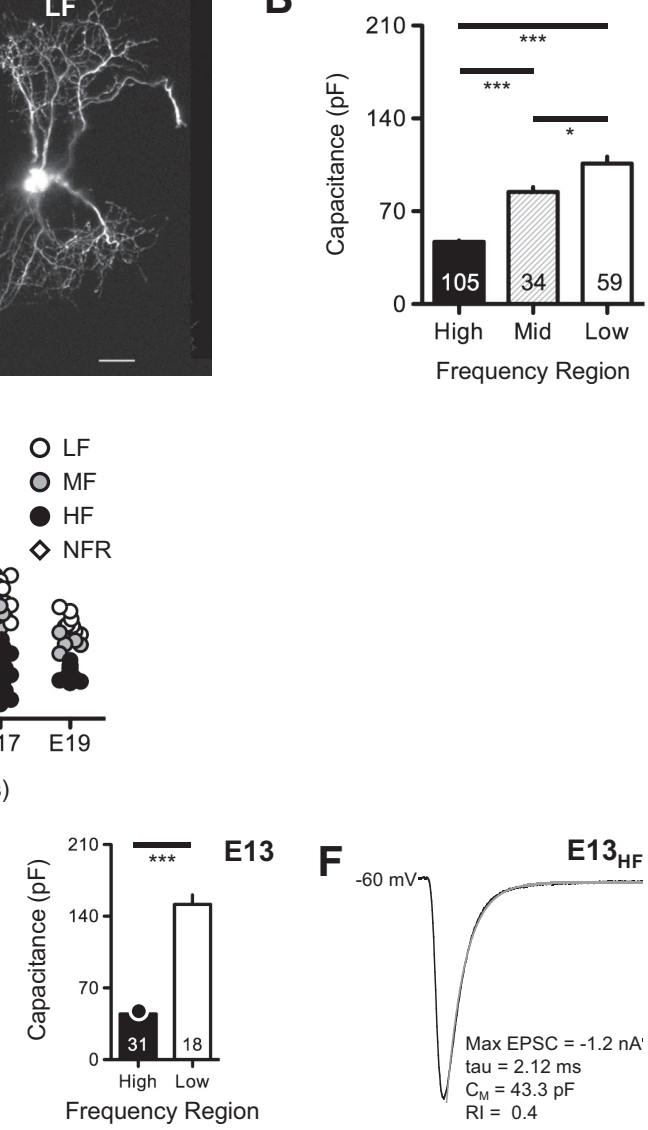

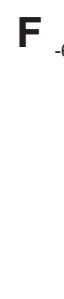

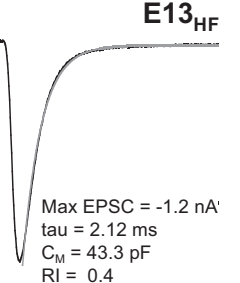

FIG. 1. Anatomical and physiological verification of recording sites along the tonotopic axis in nucleus laminaris (NL). A: dendritic gradient is parallel to the tonotopic gradient. Alexa 488 filled NL neurons from E19 tissue illustrate dendritic gradient across highmid-, and low-frequency neurons (HF, MF, and LF, respectively). $B$ : measured cell membrane capacitance $\left(C_{\mathrm{M}}\right)$ for neurons located in the $\mathrm{HF}, \mathrm{MF}$, and $\mathrm{LF}$ regions (pooled across ages). $C$ : group data summarizing differences in membrane capacitance across age and frequency regions. Increases in capacitance at E13 likely reflects an initial growth in cellular surface area, followed by pruning and thinning of dendrites at older ages (Smith 1981). NFR, no frequency region representation, diamonds at E9. D: neurobiotin labeled E13 neuron from the HF region of NL (sector 2, see METHODS). D, dorsal; M, medial. Inset: higher magnification. $E$ : $C_{\mathrm{M}}$ of E13 neurons recorded from $\mathrm{HF}$ and LF regions. Black circle in the HF bar graph represents individual data point from the neuron filled and recorded from in $D$ and $F$, respectively. Similar to pooled data across age $(B)$, neurons located in the HF region had significantly lower $C_{\mathrm{M}}$ compared with LF neurons. $F$ : evoked response in whole cell configuration from cell in $D$. Biophysical properties were similar to data reported for age-matched E13 neurons (see Figs. 3, 5, and 6). Max EPSC, maximum excitatory postsynaptic current; tau, decay time constant fit with a single exponential (superimposed gray line); RI, rectification index (see METHODS). In this and subsequent figures, traces are averaged across 20-50 stimulus presentations and stimulus artifacts removed for clarity. Numbers in bar graphs or in parentheses indicates $n$. Means \pm SE shown. Single, double, and triple asterices, $P<0.05, P<0.01, P<0.001$, respectively (1-way ANOVA, Tukey unpaired post hoc $t$-test). as described previously (Howard et al. 2007; Monsivais et al. 2000). The brain stem was dissected and isolated in ice-cold $\left(\sim 0^{\circ} \mathrm{C}\right)$ oxygenated low- $\mathrm{Ca}^{2+}$ high- $\mathrm{Mg}^{2+}$ modified artificial cerebral spinal fluid (ACSF) containing the following (in $\mathrm{mM}$ ): $130 \mathrm{NaCl}, 3 \mathrm{KCl}, 1.25 \mathrm{NaH}_{2} \mathrm{PO}_{4}, 26$ $\mathrm{NaHCO}_{3}, 4 \mathrm{MgCl}_{2}, 1 \mathrm{CaCl}_{2}$, and 10 glucose. ACSF was continuously bubbled with a mixture of $95 \% \quad \mathrm{O}_{2}-5 \% \mathrm{CO}_{2}(\mathrm{pH} 7.4$, osmolarity: 295-310 mosM/l). The brain stem was blocked coronally, affixed to the stage of a vibratome slicing chamber (Technical Products International, St. Louis, MO) and submerged in the ice-cold ACSF. Bilaterally symmetrical coronal slices were made (200-300 $\mu \mathrm{m}$ thick), and approximately one to six slices (depending on age) containing NM and NL were taken along the caudolateral to rostromedial axis, roughly representing the low- to high-frequency regions of NL, respectively.

Slices were then collected in a holding chamber and allowed to equilibrate for $1 \mathrm{~h}$ at $36^{\circ} \mathrm{C}$ in normal ACSF containing the following (in $\mathrm{mM}$ ): $130 \mathrm{NaCl}, 3 \mathrm{KCl}, 1.25 \mathrm{NaH}_{2} \mathrm{PO}_{4}, 26 \mathrm{NaHCO}_{3}, 1 \mathrm{MgCl}_{2}, 3 \mathrm{CaCl}_{2}$, and 10 glucose. Normal ACSF was continuously bubbled with a mixture of $95 \% \mathrm{O}_{2}-5 \% \mathrm{CO}_{2}$ ( $\mathrm{pH} \mathrm{7.4,} \mathrm{osmolarity:} \mathrm{295-310} \mathrm{mosM/l).} \mathrm{Slices} \mathrm{were}$ then allowed to cool to room temperature for $30 \mathrm{~min}$ before being transferred from the holding chamber to a $0.5 \mathrm{ml}$ recording chamber mounted on either a Zeiss Axioskop FS (Oberkochen, Germany) or an Olympus BX51W1 (Center Valley, PA) microscope for electrophysiological experiments. Microscopes were equipped with CCD cameras with $\times 40$ and $\times 60$ water-immersion objectives and infrared differential interference contrast optics. The recording chamber was superfused continuously with room temperature (monitored at $\sim 21^{\circ}-22^{\circ} \mathrm{C}$ ), oxygenated normal ACSF at a rate of $1.5-2 \mathrm{ml} / \mathrm{min}$.

\section{Whole cell electrophysiology}

Patch pipettes were pulled to a tip diameter of 1-2 $\mu \mathrm{m}$ that had resistances ranging from 3 to $6 \mathrm{M} \Omega$ when filled with a cesium-based internal solution containing the following (in mM): $108 \mathrm{CsMeSO}_{3}, 5$ $\mathrm{CsCl}, 1 \mathrm{MgCl}_{2}, 15$ phosphocreatine-Tris 2,8 BAPTA-Cs $_{4}$, 10 HEPES, 3 QX-314.Cl, 4 MgATP, and 0.4 Tris $_{2}$ GTP, pH adjusted to 7.3 with TrisOH. Voltage-clamp experiments were performed using either an Axoclamp 200B (Molecular Devices, Foster City, CA) or an Axon Multiclamp 700B amplifiers (Molecular Devices). For selected experiments, $2 \%$ neurobiotin was added to the internal solution for labeling of cells along the tonotopic axis (see following text). The Cs-based internal solution was used to block $\mathrm{K}^{+}$conductances and QX-314.Cl was used to block $\mathrm{Na}^{+}$conductances in an attempt to reduce spaceclamp issues associated with dendritic filtering. Command voltages during experiments were corrected for a calculated liquid junction potential of $5 \mathrm{mV}$, and series resistance was compensated for by $\sim 80 \%$ in all voltage-clamp recordings. A small hyperpolarizing $(-1$ to $-5 \mathrm{mV}, 100 \mathrm{~ms}$ ) voltage command was presented at the beginning of each recorded trace to document and monitor whole cell parameters [resting membrane potential (RMP), cell membrane capacitance, series resistance, and input resistance]. RMPs were measured immediately after break-in to avoid cesium-induced depolarization. Neurons were included in the data analysis only if they had RMPs between -50 and $-65 \mathrm{mV}$ and had series resistances $<15 \mathrm{M} \Omega$. Raw data were low-pass filtered at $2 \mathrm{kHz}$ and digitized at $10 \mathrm{kHz}$ using a data acquisition interface ITC-18 (Instrutech, Great Neck, NY) or a Digidata 1440A (Molecular Devices).

Pipettes were visually guided to NL, and neurons were identified and distinguished from surrounding tissue based on cell morphology, known laminar structure, and location of the nucleus within the slice. After a $G \Omega$ seal was attained, membrane patches were ruptured and NL neurons were held in whole cell configuration at $-60 \mathrm{mV}$ for recording of isolated AMPA-R mediated excitatory postsynaptic currents (EPSCs) or at $+40 \mathrm{mV}$ for recording of isolated NMDA-R mediated EPSCs. Isolated AMPA-R currents were recorded in the 
presence of an NMDA-R blocker D-2-amino-5-phosphonopentanoic acid (D-APV, $100 \mu \mathrm{M}$ ), and isolated NMDA-R currents were recorded in the presence of AMPA-R blockers 1,2,3,4-tetrahydro-6-nitro-2,3-dioxo-benzo[f]quinoxaline-7-sulfonamide disodium salt hydrate (NBQX, $20 \mu \mathrm{M})$ or 6,7-dinitroquinoxaline-2,3(1H,4H)-dione (DNQX, $50 \mu \mathrm{M})$. All experiments were conducted in the presence of $\mathrm{GABA}_{\mathrm{A}}-\mathrm{R}$ blockers bicuculine methiodide (BIC, $50 \mu \mathrm{M}$ ), SR-95331 (gabazine, $10 \mu \mathrm{M}$ ), or picrotoxin (PTX, $100 \mu \mathrm{M})$.

Extracellular synaptic stimulation was accomplished using a concentric bipolar electrode (tip core diameter $=200 \mu \mathrm{m}$, World Precision Instruments, Sarasota, FL). Square electric pulses, $100 \mu \mathrm{s}$ in duration, were delivered by a stimulus isolator (1850A) and interval generator (1830; WP Instruments, New Haven, CT) or by an Iso-flux stimulator (AMPI; Jerusalem, Israel) and interval generator (S88; Grass, West Warwick, RI). Stimulating electrodes were placed in either the ventral or dorsal neuropil region of NL, $\sim 30-50 \mu \mathrm{m}$ from recorded NL neurons. No differences were observed in the size of maximum ESPC amplitudes or kinetics based on stimulating electrode placements (dorsal versus ventral). Stimulation current was adjusted from 0 to $200 \mu \mathrm{A}$ in steps of 5-10 $\mu \mathrm{A}$. Input-output functions were derived for each NL neuron and the stimulus intensity was adjusted to evoke the maximum EPSC, often resulting in a plateau EPSC response, in an attempt to recruit every and all-excitatory inputs from one side of the brain. This stimulus intensity $($ mean $=163 \pm 4.8 \mu \mathrm{A})$ never exceeded the output of the stimulus generator and was $20 \%$ less than the maximal intensity tolerated in this slice preparation without causing hydrolysis or motion of the stimulating electrode tip.

The AMPA-R agonist 2-carboxy-3-carboxymethyl-4-isopropenylpyrrolidine (kainate, $100 \mu \mathrm{M}$ ) and the NMDA-R agonist $(R)-2$ (methylamino) succinic acid (NMDA, $500 \mu \mathrm{M}$ ) were puff applied with a picosprtizer (General Valve, Fairfield, NJ). Puff pipettes were pulled to a tip diameter of 3-6 $\mu \mathrm{m}$ and filled with ACSF containing either kainate or NMDA. Puff pipettes were visually guided into close proximity $(\sim 20-40 \mu \mathrm{m})$ of the neuron from which recordings were being made. Pressure pulses used (10-20 psi; 20-50 ms duration) did not damage neurons or disrupt patch-pipette seals. Isolated AMPA-R mediated miniature EPSCs (mEPSCs) were recorded with tetrodotoxin (TTX, $1 \mu \mathrm{M})$ and D-APV added to the external bath ACSF solution. For experiments where $I-V$ relationships were constructed for isolated AMPA-R current, N,N-bis(3-aminopropyl)-1,4-diaminobutane, gerontine, musculamine, neuridine (spermine, $100 \mu \mathrm{M}$ ), a polyamine ion channel blocker for AMPA-Rs lacking the GluR2 subunit, was added to the internal pipette solution to reduce dialyzing endogenous polyamines. To accurately report the NMDA-R decay time constant (tau), a weighted tau was calculated as previously described (Rumbaugh and Vicini 1999) using the following formula: $\tau_{\mathrm{W}}=\tau_{\mathrm{F}}\left[I_{\mathrm{F}} /\left(I_{\mathrm{F}}+I_{\mathrm{S}}\right)\right]+\tau_{\mathrm{S}}\left[I_{\mathrm{S}}\left(I_{\mathrm{F}}+I_{\mathrm{S}}\right)\right]$, where $I$ is the current amplitude, $I_{\mathrm{F}}$ and $I_{\mathrm{S}}$ are the peak amplitudes of the fast and slow components, respectively, and $\tau_{\mathrm{F}}$ and $\tau_{\mathrm{S}}$ are the respective time constants.

\section{$\mathrm{Co}^{2+}$ labeling of GluR2-lacking AMPA-Rs}

Procedures for $\mathrm{Co}^{2+}$ uptake studies were modified from previous reports (Estabel et al. 1999; Pruss et al. 1991; Zhou et al. 1995). Coronal slices $(500-\mu \mathrm{m}$-thick) through NL were prepared from E11, E15, E19, and E21 embryos and P5 hatchlings as described in the preceding text and collected in oxygenated ACSF. Slices were preincubated in $5 \mathrm{mM} \mathrm{CoCl}_{2}$ for 20 min before the addition of kainate (100 $\mu \mathrm{M}$; Sigma, St. Louis, MO) for another $20 \mathrm{~min}$. For control cases, slices were either incubated in $\mathrm{CoCl}_{2}$ only or incubated in both $\mathrm{CoCl}_{2}$ and Kainate and $50 \mu \mathrm{M}$ DNQX. Slices were then washed in ACSF for $5 \mathrm{~min}$. Extracellular cobalt was removed by a 10-min incubation in 2 mM EDTA to reduce background. Slices were washed twice in ACSF each for $5 \mathrm{~min}$ before being developed in 1.2\% (wt/vol) ammonium sulfide for $10 \mathrm{~min}$. After two 5-min washes in ACSF, slices were fixed in $4 \%$ paraformaldehyde in $0.1 \mathrm{M}$ phosphate buffer for 2-5 h. Slices were cryoprotected in $30 \%$ sucrose in $0.1 \mathrm{M}$ phosphate buffer overnight and then resectioned at $30 \mu \mathrm{m}$ on a freezing sliding microtome. Sections were mounted on gelatin-coated slides and then dehydrated, cleared, and coverslipped with DPX mounting medium (EMS, Hatfield, PA).

Digital images of selected sections with cobalt staining were captured with a Zeiss AxioPlan 2ie equipped with a Coolsnap HQ monochrome digital camera (Princeton Instruments, Trenton, NJ) through a No. 22 Wratten filter and collected in Slidebook (version 4.0.2.8; Intelligent Imaging Innovations, Denver, CO). Image contrast and brightness adjustments were made in Adobe Photoshop (Adobe Systems, Mountain View, CA).

\section{Anatomical verification of recorded neurons along the tonotopic gradient in $N L$}

Characteristic frequencies (CFs) of NL neurons increase linearly from the caudolateral to the rostromedial pole (Parks and Rubel 1975). To verify the anatomical region of NL recordings, one to six coronal slices containing the entire extent of NL were obtained from each animal. The number of slices was dependent on the age of the animal (see following text). The level of each slice was confirmed by the arrangement of NL and adjacent NM. Anatomical verification of recorded neurons along the tonotopic gradient in NL was determined based on previous studies (Parks and Rubel 1975, 1978; Smith and Rubel 1979; Smith 1981). In addition to recording the rostrocaudal position, each coronal slice was divided into one to four sectors, depending on their mediolateral position within NL (Kuba et al. 2005). This procedure allowed us to define $\sim 11$ sectors for NL in each preparation for animals E13-E19. Using this procedure, three CF regions were defined as follows: rostral sectors $1-4$ as the highfrequency (HF) region, intermediate sectors $5-8$ as the middlefrequency (MF) region, and caudal sectors 9-11 as the low-frequency (LF) region. This corresponds to predicted CFs of $2.5-3.3 \mathrm{kHz}$ for the $\mathrm{HF}$ region, $1-2.5 \mathrm{kHz}$ for the MF region, and $0.4-1 \mathrm{kHz}$ for the LF region, respectively (Kuba et al. 2005; Parks and Rubel 1975, 1978). However, due to the small size of embryonic brain stem tissue at E9, we obtained only one to two slices per animal, and no tonotopic information was gathered at this age. Similarly, only two slices were consistently obtained for E11 tissue. As a result, six sectors were used to define only the HF and LF tonotopic regions at E11. Rostral slices were divided into sectors 1-3 (HF regions) while caudal slices were divided into sectors 4-6 (LF regions). In addition, cell membrane capacitance $\left(C_{\mathrm{M}}\right)$ was determined for each neuron within a given sector to further correlate neuron size with tonotopic location with the assumption that HF neurons would have smaller $C_{\mathrm{M}}$ compared with LF neurons based on dendritic size. $C_{\mathrm{M}}$ was calculated based on a steady-state current response elicited by a small hyperpolarizing voltage command ( -1 to $-5 \mathrm{mV}$ ) after whole cell capacitance compensation. Neurons recorded from the LF region (sectors 9-11) of NL had significantly larger $C_{\mathrm{M}}$ than MF and $\mathrm{HF}$ neurons (sectors $1-4$, and 5-8, respectively, $P<0.05$, Fig. $1 B$ ). This result was consistent across developmental ages (Fig. 1C). To further confirm recorded position from individual NL neurons, $2 \%$ neurobiotin was included in the recording pipette solution to visualize dendritic length along tonotopic regions of NL. Slices that contain labeled neurons were fixed by immersion in $4 \%$ paraformaldehyde in $\mathrm{PB}$ overnight and visualized with 3-3'-diaminobenzidine (DAB). An example of an AMPA-R mediated EPSC recorded from a neurobiotin labeled E13 HF neuron (from sector 2) is shown in Fig. 1, $D-F$. The single cell body layer and neuropil region of the nucleus is identifiable and the short bitufted dendrites of the labeled NL neuron are visible (Fig. $1 D$ ). The measured $C_{\mathrm{M}}$ for this neuron was significantly smaller than LF neurons recorded from the same developmental age (Fig. 1E). Additionally, the physiologic response properties (i.e., maximum EPSC amplitude, decay tau, and rectification index) of this neuron are representative of an E13 HF neuron (Fig. $1 F$, compare with Figs. 3, 5, and 6). 


\section{Data analysis}

Recording protocols were written and run using either the Axograph acquisition and analysis software (version 4.5; Molecular Devices) or the Clampex acquisition and Clampfit analysis software (version 10.1; Molecular Devices). mEPSCs were detected using a template, the kinetics (i.e., 10-90\% rise-time and decay time constant) of which resembled that of a typical AMPA-R mediated mEPSC. A detection threshold was set at $5 \mathrm{pA}$, and all events were analyzed using mini-analysis software (Synaptosoft, Fort Lee, NJ). Statistical analyses (ANOVAs, post hoc test, $t$-test, regression analysis) and graphing protocols were performed using Prism (GraphPad versions 5.0a, La Jolla, CA). The standard for significant differences was defined as $P<0.05$. All graphic representations of data illustrate means \pm SE. Tables represent means \pm SD. The number of observations $(n)$ contributing to each mean is reported in parentheses or inside bar graphs for each figure.

\section{Reagents}

All bath applied drugs were allowed to perfuse through the recording chamber for $\sim 2$ min before subsequent recordings. D-APV, NBQX, DNQX, kainate, NMDA, BIC, gabazine, spermine, and all other salts and chemicals were obtained from Sigma-Aldrich (St. Louis, MO). $N, N, N$,rimethyl-5-[(tricyclo[3.3.1.13,7]dec-1-ylmethyl)amino]-1-pentanaminiumbromide hydrobromide (IEM-1460, $100 \mu \mathrm{M})$, (aR,bS)-a-(4hydroxyphenyl)-b-methyl-4-(phenylmethyl)-1-piperidinepropanol maleate (Ro25-6981, $1 \mu \mathrm{M}$ ), and PTX were obtained from Tocris (Ellisville, MO). TTX and QX-314 were obtained from Alomone Labs (Jerusalem, Israel).

\section{R E S U L T S}

We characterized the biophysical properties of AMPA-R and NMDA-R mediated EPSCs across developmental age and tonotopic regions in NL. A total of $208 \mathrm{NL}$ neurons were recorded from slice preparations taken at E9 $(n=6), \mathrm{E} 10(n=$ $4), \mathrm{E} 11(n=27), \mathrm{E} 13(n=62), \mathrm{E} 15(n=34), \mathrm{E} 17(n=45)$, and E19 $(n=30)$ chicken embryos. We describe dramatic changes in synaptic responses mediated by iGluRs during a time period when hearing is first emerging and dendritic morphology is being established across the tonotopic gradient.

\section{Onset of iGluR responses}

We observe physiologically functional iGluRs as early as NL neurons were reliably identifiable in brain stem slices (E9). Interestingly, the presence of iGluRs is evident as early as E7 in nucleus magnocellularis (NM) (Diaz et al. 2009), suggesting that iGluRs are present in both NM and NL prior to synaptogenesis. At E9 in NL, relative dendritic homogeneity is observed across different tonotopic regions (Smith and Rubel 1979; Smith 1981) and postsynaptic action potentials are not evoked by stimulation of afferent inputs from NM axons (Jackson et al. 1982). Puff application of an NMDA-R agonist (NMDA, $500 \mu \mathrm{M}$ ), while voltage clamping NL neurons at +40 $\mathrm{mV}$, elicited a robust outward current that was reversibly blocked by bath application of the NMDA-R antagonist D-APV (Fig. 2A1). Likewise, puff application of an AMPA-R agonist (kainate, $100 \mu \mathrm{M}$ ), while voltage clamping E9 NL neurons at $-60 \mathrm{mV}$, elicited a strong inward current that was susceptible to bath application of the AMPA-R antagonist NBQX (Fig. 2A2). These robust iGluR currents to puff applications of agonists were always observed, while electrical stimulation of afferent excitatory inputs from NM never elicited an EPSC in all neurons tested at E9 $(n=6)$, consistent with observations previously reported (Jackson et al. 1982). In contrast, synaptically evoked iGluR-mediated EPSCs were present $\sim 75 \%$ of the time at E10 (data not shown). These results indicate that both AMPA-Rs and NMDA-Rs are functionally present $\geq 1$ day before synaptic contacts are established.

\section{Changes in synaptic iGluR responses}

At E11, 1 day after synaptogenesis, evoked EPSCs exhibited two clearly discernible peaks when the voltage of the neuron was held at $+40 \mathrm{mV}$ (Fig. 2Bl). These dual-component EPSCs contained both AMPA-R and NMDA-R mediated currents that could be pharmacologically isolated. Bath application of the NMDA-R antagonist D-APV eliminated the slower response leaving an early and faster component mediated by the AMPA-R. Subsequent bath application of NBQX completely eliminated the remaining AMPA-R response (Fig. $2 B 1)$. The peak amplitude ratio of the slow EPSC component (NMDA-R mediated) to the fast EPSC component (AMPA-R mediated) measured at $+40 \mathrm{mV}$, resulted in an NMDA/AMPA ratio close to $1(0.96 \pm 0.04 ; n=4)$. It should be noted that in our experiments at E11, we never observed NMDA-R only EPSCs (i.e., silent synapses). This suggests that synaptogenesis in NL is initiated with both iGluRs present, similar to what has been described for developing NM (Lu and Trussell 2007), and is in contrast to observations made at other developing central synapses (Durand et al. 1996; Isaac et al. 1997; Liao et al. 1995; Rumpel et al. 2004).

The ratio of isolated NMDA-R responses recorded at $+40 \mathrm{mV}$ to isolated AMPA-R responses recorded at $-60 \mathrm{mV}$ (Fig. $2 B, 2$ and 3) showed a similar value as the ratio of the two peaks observed at $+40 \mathrm{mV}(0.94 \pm 0.08 ; n=11)$. These results suggest that AMPA-Rs do not rectify at E11 and that NMDA-R mediated currents are of similar magnitude as AMPA-R mediated currents. In contrast, at E19, when synaptic responses are considered to be nearly mature (Kuba et al. 2002a), a significant difference in the contribution of AMPA-Rs and NMDA-Rs is clearly observed. The NMDA/AMPA ratio recorded at $+40 \mathrm{mV}$ was $0.28 \pm 0.10$, largely due to an increase in the AMPA-R component with little change in the NMDA-R response (Fig. $2 \mathrm{Cl}$; note different scale bar values). Moreover, the ratio of isolated NMDA-R responses recorded at $+40 \mathrm{mV}$ to isolated AMPA-R responses recorded at $-60 \mathrm{mV}$ was $0.04 \pm 0.03$ due to an even larger AMPA-R mediated current observed at $-60 \mathrm{mV}$ (Fig. 2C, 2 and 3). This difference in the amplitude of the AMPA-R current at hyperpolarized and depolarized membrane potentials suggest that at older ages the AMPA-R rectifies.

These data indicate that, initially, currents mediated by AMPA-Rs and NMDA-Rs are comparable (Fig. 2B, 1-3). However, the contribution of each receptor changes dramatically within a few days. The AMPA-R current increases significantly (Fig. 2, $B 2$ and $C 2$, note different scale bars), a classic indicator of synaptic maturity (Carmignoto and Vicini 1992; Hestrin 1992), while the NMDA-R current peaks at E17 (see Fig. 8), then is reduced at E19 to a similar level as E11 (Figs. 2, B3 and C3, and 8). Specific changes in both AMPA-R and NMDA-R responses across age and tonotopic regions are discussed in greater detail next. 
$A_{1}$

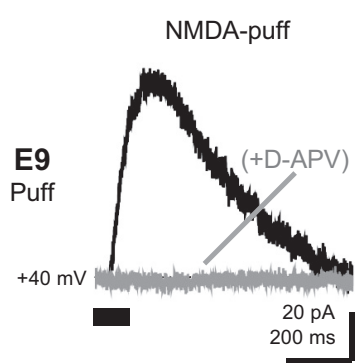

$B_{1}$

Dual-component EPSC

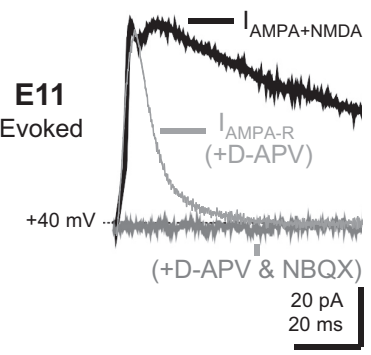

$C_{1}$

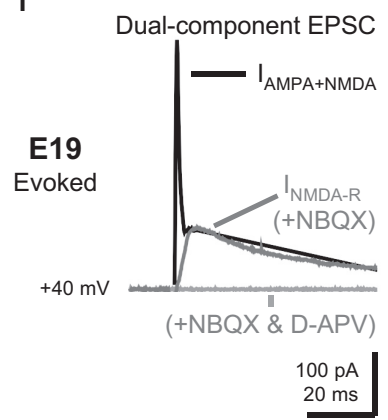

$\mathrm{A}_{2}$

Kainate-puff

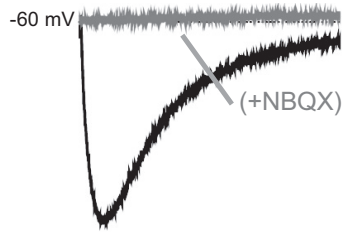

$20 \mathrm{pA}$

$\mathrm{B}_{2}$

Isolated AMPA-R EPSC

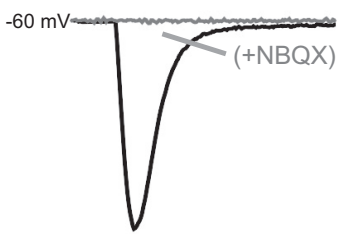

$20 \mathrm{pA}$

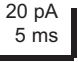

$\mathrm{C}_{2}$

Isolated AMPA-R EPSC
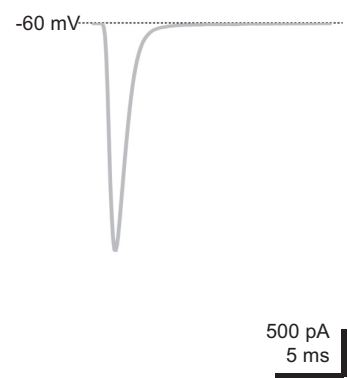

$B_{3}$

Isolated NMDA-R EPSC
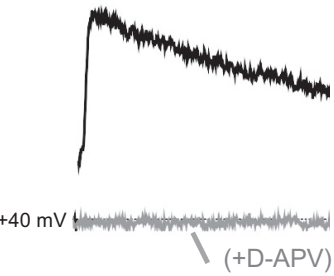

$20 \mathrm{pA}$

$\mathrm{C}_{3}$

Isolated NMDA-R EPSC

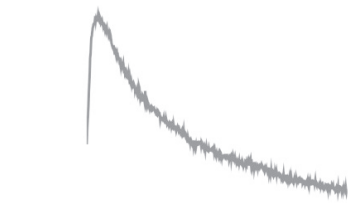

$+40 \mathrm{mV}$

$20 \mathrm{pA}$

FIG. 2. Developmental changes in ionotropic glutamate receptor (iGluR) currents. Example traces of whole cell recordings from E9, E11, and E19 NL neurons. Recovery traces not shown for clarity. A1: puff application of $N$-methyl-D-aspartate (NMDA, $500 \mu \mathrm{M}$, bar) at E9 elicited an outward current (black trace; voltage-clamp $=+40 \mathrm{mV}$ ) that was blocked by D-2-amino-5-phosphonopentanoic acid (D-APV, $100 \mu \mathrm{M}$, gray trace). A2: puff application of kainate (100 $\mu \mathrm{M}$, bar) at E9 elicited an inward current (black trace; voltage clamp $=-60 \mathrm{mV}$ ) that was blocked by 1,2,3,4-tetrahydro-6-nitro-2,3-dioxo-benzo[f]quinoxaline7-sulfonamide disodium salt hydrate (NBQX, $20 \mu \mathrm{M}$, gray trace). B1: EPSCs are mediated by AMPA and NMDA receptors (AMPA-Rs and NMDA-Rs) at E11. Stimulation of afferent fibers with a bipolar electrode (see METHODS) produced a dual component EPSC ( $I_{\text {AMPA+NMDA }}$, black trace). Application of D-APV eliminated the late response leaving an early component mediated by the AMPA-R ( $I_{\text {AMPA-R }}$, gray trace). Subsequent application of NBQX completely eliminated the AMPA-R response (thick gray trace). B, 2 and 3: isolated AMPA-R and NMDA-R mediated EPSCs at E11 (black traces in $B, 2$ and 3, respectively) were reversibly blocked by bath application of their respected antagonist (NBQX and D-APV; gray traces). C1: EPSCs are mediated by AMPA-Rs and NMDA-Rs at E19. Dual-component EPSCs recorded as in $B$ ( $I_{\mathrm{AMPA}+\mathrm{NMDA}}$, black trace). Application of NBQX eliminated the early response leaving a late component mediated by the NMDA-R ( $I_{\text {NMDA-R, }}$ gray trace). Subsequent application of D-APV completely eliminated the NMDA-R response (light gray trace). $C$, 2 and 3: isolated AMPA-R and NMDA-R mediated EPSCs at E19. The AMPA-R current at E19 (gray trace in C2) was 10-fold larger than the AMPA-R current at E11 (black trace in B2; note different scale bar values), while the NMDA-R current at E19 (gray trace in C3) was nearly equal in size compared with the NMDA-R current at E11 (black trace in B3). Dash lines in $C, 2$ and 3, represent baseline.

\section{Increase in the amplitude of evoked AMPA-R} mediated EPSCs

To study changes in the amplitude of evoked glutamatergic EPSCs across different embryonic days and tonotopic regions, a maximal stimulation protocol was used to allow comparisons across tonotopic regions within an age and differences across age in the same tonotopic region. Input-output $(\mathrm{I} / \mathrm{O})$ functions were determined for each NL neuron until a plateau was reached (Fig. 3A), indicating that most, if not all, excitatory inputs from one NM have been recruited (see METHODS). The stimulus intensity chosen to evoke maximum AMPA-R currents was determined from individual I/O curves such that the stimulation gave a reliable maximum response. The example in Fig. $3 A$ shows an E13 neuron recorded from the HF region of NL. Note that increases in stimulus intensity resulted in stepwise increase in amplitude of EPSCs. We also observed no failures at minimal stimulation levels, consistent with a high probability of release. The arrow in Fig. $3 A$ indicates the intensity used to obtain a maximum response. This stimulus intensity never exceeded the output of the stimulus generator. When recorded in this fashion, neurons from the HF region of NL showed a significant increase in the amplitude of maximally evoked AMPA-R mediated EPSCs with age. Example traces of maximum AMPA-R responses recorded from the HF region of E13, E15, and E17 NL neurons 
A

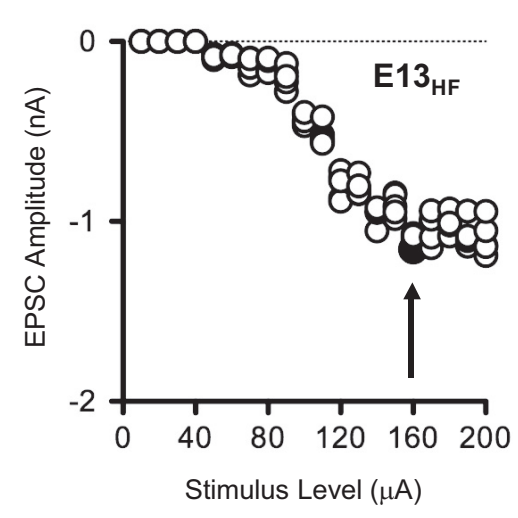

D

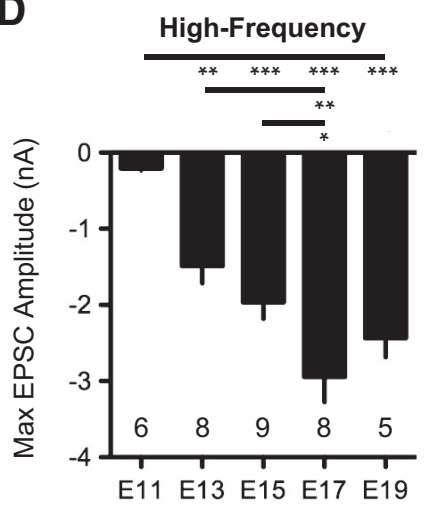

B

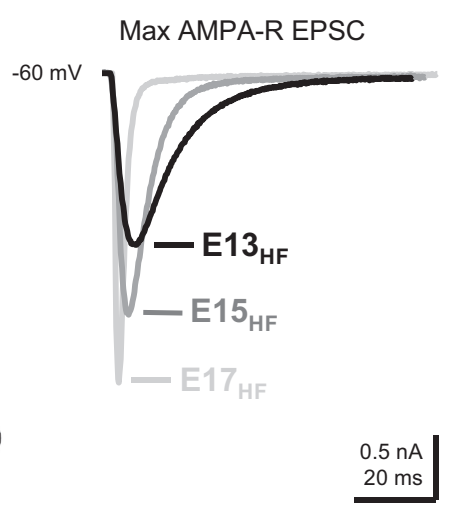

C

$-60 \mathrm{mV}$

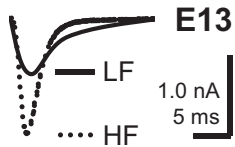

$-60 \mathrm{mV}$

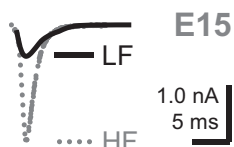

$-60 \mathrm{mV}$

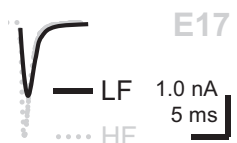

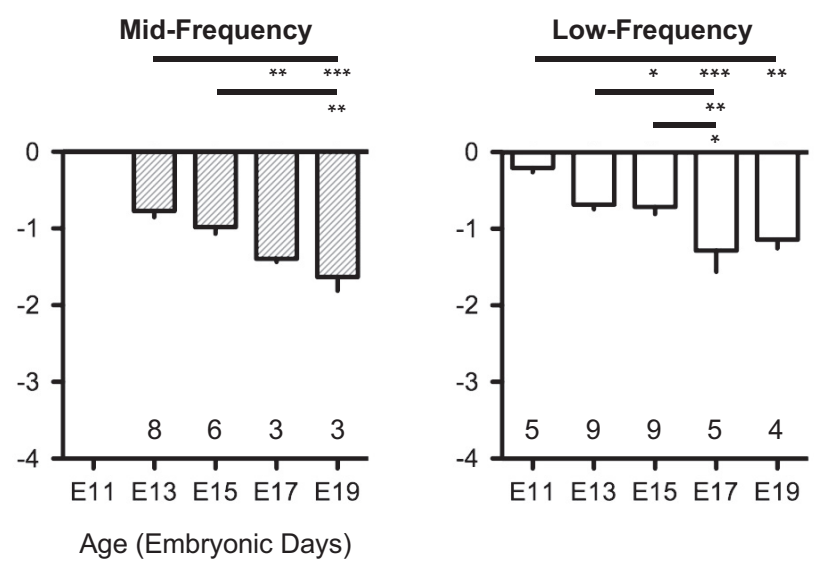

FIG. 3. Isolated AMPA-R currents increase with age and across tonotopic regions. A: isolated AMPA-R mediated EPSCs plotted as a function of stimulus intensity

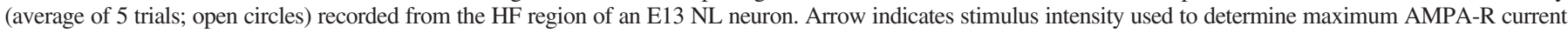

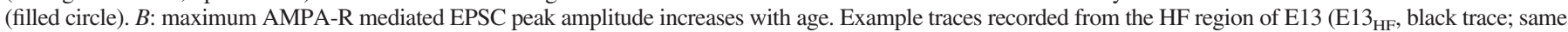

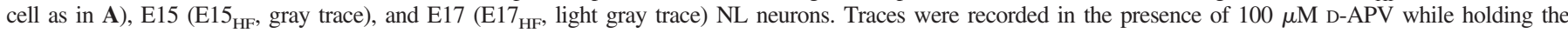

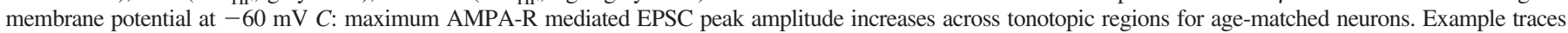

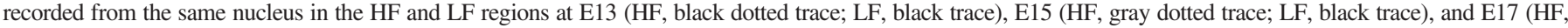
light gray dotted trace; LF, black trace). D: population data of maximum AMPA-R mediated EPSCs from the HF, MF, and LF regions.

are shown in Fig. 3B. For HF neurons, AMPA-R responses increased 14-fold from E11 to E17. This profile was similaralbeit with less degree of change-at different tonotopic regions of NL. That is, MF neurons resulted in a modest yet significant twofold increase from E13 to E17, and LF neurons resulted in a sixfold increase from E11 to E17. Significant changes in maximum amplitude were not seen after E17. Similar results were obtained using a minimal stimulation protocol of afferent inputs from NM as determined by individual I/O functions (data not shown), further supporting the results that the amplitude of AMPA-R mediated EPSCs increased from E11 to E17, becoming relatively stable by E19.

Neurons recorded from the HF region of NL always had larger EPSCs compared with age-matched neurons recorded from $\mathrm{LF}$ regions. Example traces recorded from the $\mathrm{HF}$ and $\mathrm{LF}$ regions from the same NL at E13, E15, and E17 are shown in Fig. 3C. Similar results were obtained using a minimal stimulation protocol. Population data are summarized in Fig. $3 D$ and show significant increases in the maximum AMPA-R response with age and across tonotopic regions in NL.

Although we cannot rule out the possibility of space-clamp issues associated with dendritic filtering as a possible explanation for the decrease in EPSC amplitude across different tonotopic regions (there is nearly a 10 -fold gradient in dendritic length that runs parallel to the tonotopic axis), we believe that this is not the case. The rise time of EPSCs, measured at $10-90 \%$, were rapid at all ages (Table 1, pooled average $=$ $0.82 \pm 0.21 \mathrm{~ms}, n=88$ ), and differences in rise times across

TABLE 1. AMPA-R rise time across development and tonotopic regions in NL

\begin{tabular}{lcccrr}
\hline \hline & E11 & E13 & E15 & E17 & E19 \\
\hline HF & $1.18 \pm 0.25(6)$ & $0.81 \pm 0.27(8)$ & $0.71 \pm 0.18(9)$ & $0.59 \pm 0.19(8)$ & $0.47 \pm 0.05(5)$ \\
MF & & $1.07 \pm 0.19(8)$ & $0.83 \pm 0.21(6)$ & $0.77 \pm 0.09(3)$ & $0.52 \pm 0.11(3)$ \\
LF & $0.95 \pm 0.22(5)$ & $1.07 \pm 0.36(9)$ & $0.98 \pm 0.31(9)$ & $0.89 \pm 0.29(5)$ & $0.66 \pm 0.19(4)$ \\
\hline
\end{tabular}

Mean \pm SD is shown. HF, MF, and LF represent information from the high-, mid-, and low-frequency regions of nucleus laminaris (NL), respectively. Number of neurons indicated in parentheses. 
tonotopic regions were not significant at any age. Moreover, there is no correlation in amplitude and rise time at any age (pooled correlation coefficient $=0.24, n=88$ ). In addition, we routinely monitored the reversal potential of AMPA-R currents. Only neurons with reversals potential near $0 \mathrm{mV}$ were used in the analysis. Further evidence against dendritic filtering is discussed in upcoming sections. It should also be noted that we were unable to reliably record MF neurons from E11 NL slices due to the relatively small nucleus compared with E13 and older tissue. Therefore only neurons sampled from the rostromedial and caudolateral regions of the nucleus were used at E11, representing $\mathrm{HF}$ and LF neurons, respectively (see METHODS).

\section{Increase in the frequency of spontaneous AMPA-R mediated} miniature EPSCS

The increase in amplitude of evoked AMPA-R mediated EPSCs suggests several possibilities: an increase in the number of synaptic contacts, an increase in density of synaptic AMPARs, or changes that would affect release probability, i.e., an increase in presynaptic $\mathrm{Ca}^{2+}$ channels. To test some of these possibilities, we recorded spontaneous miniature AMPA-R mediated EPSCs (mEPSCs) in the presence of the $\mathrm{Na}^{2+}$. channel blocker TTX and the NMDA-R antagonist D-APV. A large increase in the frequency of mEPSCs is observed through development (Fig. 4 and Table 2), suggesting that an increase in synaptic contacts account for the increase in evoked EPSCs observed (Hsia et al. 1998; Petralia et al. 1999). Example traces are shown in Fig. $4 A$ from E13 and E17 NL neurons recorded from the $\mathrm{HF}$ and $\mathrm{LF}$ regions. The inter-event interval significantly decreased with age and across tonotopic regions, indicating an increase in the frequency of spontaneous events. The highest frequency was observed for E17 neurons from the HF region (Fig. 4, $A$ and $B$ ). As expected for an increase in frequency, the total number of events increased with age and across different tonotopic regions, with E17 neurons from the $\mathrm{HF}$ region having the most events per neuron (Fig. $4 \mathrm{C}$ and Table 2). Thus the overall mEPSC frequency increased $\sim 10$ fold from E13 to E17, a slightly smaller increase than that of the maximum amplitude of the evoked EPSC for the same age period.

The mEPSCs $10-90 \%$ rise times were quite rapid and differences in rise times across age and tonotopic regions were not significant (Fig. $4 D$ and Table 2, $P=0.89$ ). Surprisingly, differences in decay tau across age and tonotopic regions were also not significant despite a twofold increase in decay tau across the same developmental time period of evoked EPSCs (Fig. $4 D, P=0.57$, see next section). There was no correlation between mEPSC amplitude and rise time at any age (pooled correlation coefficient $=0.04$ ), suggesting that dendrites did not compromise the control of membrane voltages despite differences in dendritic length across tonotopic regions of NL. In addition, the amplitude of mEPSCs did not significantly change across ages (Fig. $4 D$ and Table 2, $P=0.92$ ), and the population data showed stable amplitude histograms across age and tonotopic region (Fig. $4 C$ ).

To probe for changes in release probability, we evoked pairs of EPSCs at $100 \mathrm{~ms}$ apart. Strong paired pulse synaptic depression was observed as early as E13 (Fig. 4E) and changes in the paired-pulse ratio (2nd EPSC/1st EPSC) did

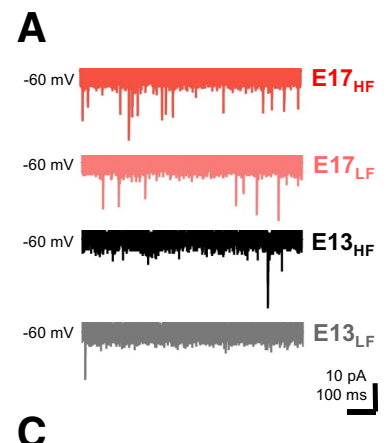

B
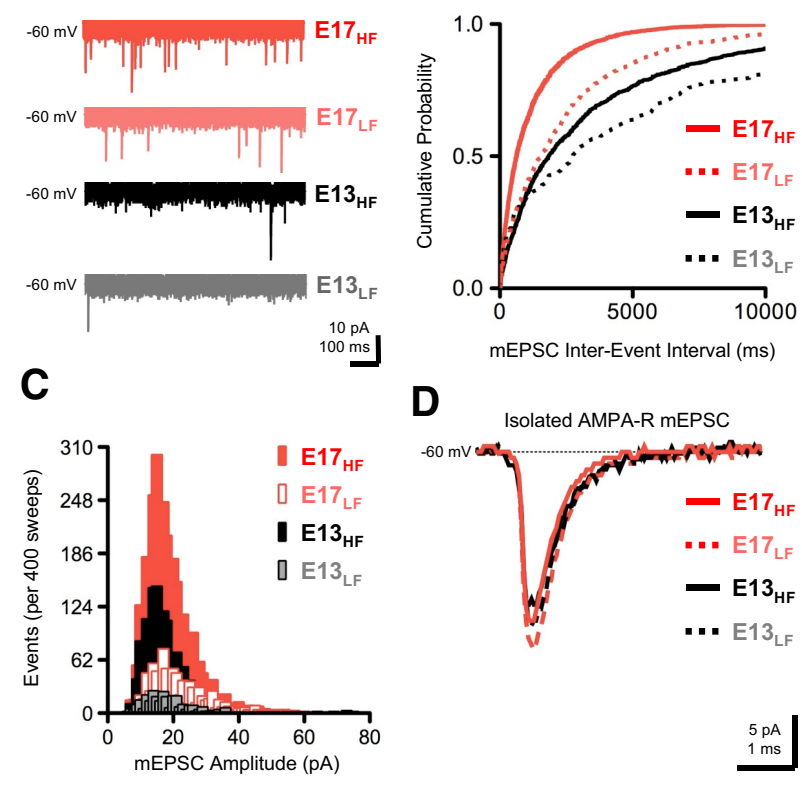

mEPSC Inter-Event Interval (ms)
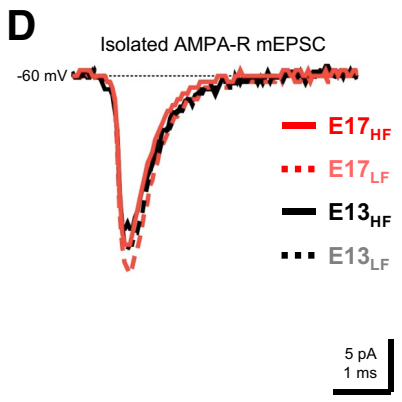

E

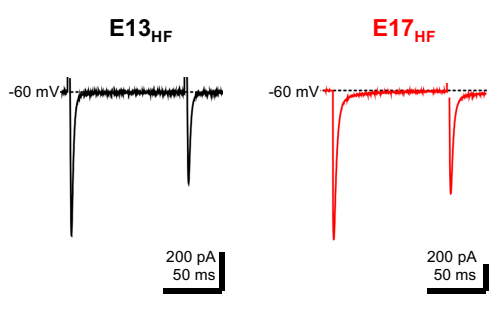

$\mathbf{F}$

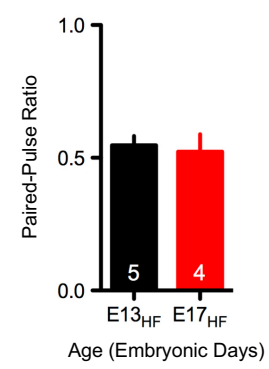

FIG. 4. Frequency of isolated AMPA-R miniature EPSCs (mEPSCs) increase with age and across tonotopic regions. A: representative AMPA-R mediated mEPSCs recorded from E17 and E13 NL neurons located in the HF region $\left(\mathrm{E} 17_{\mathrm{HF}}\right.$, red trace; $\mathrm{E} 13_{\mathrm{HF}}$, black trace) and $\mathrm{LF}$ region $\left(\mathrm{E} 17_{\mathrm{LF}}\right.$, pink trace; E13 ${ }_{\mathrm{LF}}$, gray trace) of the same NL. Spontaneous mEPSCs were recorded in the presence of $50 \mu \mathrm{M}$ TTX and $100 \mu \mathrm{M}$ D-APV. $B$ : cumulative probability plot shows a significant increase in the inter-event interval of mEPSCs across age and tonotopic region. $C$ : population data from the HF and LF regions of E17 and E13 NL neurons. Histogram shows similar amplitude distribution across age and tonotopic region. $n=8$ neurons for $\mathrm{E} 17_{\mathrm{HF}}$ (red bars; total events = 2992); 5 neurons for E17 $\mathrm{LF}$ (white bars; total events $=878$ ), 12 neurons for E13 ${ }_{\mathrm{HF}}$ (black bars; total events $=1452$ ), and 4 neurons for $\mathrm{E} 13_{\mathrm{LF}}$ (gray bars; total events $=303)$. $D$ : average traces from $\mathrm{E} 17_{\mathrm{HF}}$ (red solid line, events $=$ 374), E17 $7_{\mathrm{LF}}$ (red dotted line, events $\left.=176\right), \mathrm{E} 13_{\mathrm{HF}}$ (black solid line, events $=$ 121 ), and E13 $3_{\mathrm{LF}}$ (black dotted line, events $=76$ ) show similar AMPA-R mediated mEPSC amplitudes and kinetics (same neurons in $A$ ). $E$ : paired pulse recordings (100 ms interval) from the HF region of E13 (left, black trace) and E17 (right, red trace) NL neurons. $F$ : population data showing paired pulse ratios (pulse $2_{\text {EPSC }}$ /pulse $1_{\text {EPSC }}$ ) for $\mathrm{HF}$ neurons recorded at E13 (black bar) and E17 (red bar).

not decrease with age (Fig. 4, E and F), suggesting that the probability of release remains constant throughout this developmental period. In addition, we observed a low coefficient of variation (CV) in the amplitude of evoked EPSCs that remained constant across development. The data indicate that no changes in the probability of release occur during this development period.

Although we cannot rule out an increase in receptor density, the large increase in mEPSC frequency is sufficient to account for the large increase in AMPA-R synaptic transmission. Our results suggest that older neurons located in the $\mathrm{HF}$ region 
TABLE 2. Summary of numerical data on AMPA-R miniature excitatory postsynaptic currents across specific developmental periods and tonotopic regions in $N L$

\begin{tabular}{ccccc}
\hline \hline & Events* & Amplitude, pA & Rise, ms & Decay, ms \\
\hline E13 & & & & \\
HF & $121 \pm 61(12)$ & $-18.11 \pm 8.19(12)$ & $0.38 \pm 0.04(12)$ & $0.99 \pm 0.37(12)$ \\
LF & $76 \pm 47(4)$ & $-19.32 \pm 8.12(4)$ & & $1.02 \pm 0.86(4)$ \\
E17 & & & $0.38 \pm 0.03(8)$ & $0.88 \pm 0.43(8)$ \\
HF & $374 \pm 12(8)$ & $-19.78 \pm 8.21(8)$ & $0.39 \pm 0.02(5)$ & $0.99 \pm 0.40(5)$ \\
LF & $176 \pm 33(5)$ & $-23.20 \pm 10.11(5)$ & \\
\hline
\end{tabular}

Decay time constant (tau) fit with a single exponential. Number of neurons indicated in parentheses. *Averaged number of events per neurons.

contain more synaptic contacts than younger neurons located in the LF region of NL despite HF neurons being considerably smaller than LF neurons.

\section{Changes in AMPA-R mediated EPSC kinetics}

In several brain regions, the AMPA-R component of evoked EPSCs shows striking developmental changes in kinetics, an effect accounted for by alterations in the AMPA-R subunit composition (Dingledine et al. 1999; Lu and Trussell 2007; Sabatini and Regehr 1999). We also observe a clear acceleration in the AMPA-R mediated EPSC decay tau during development and across different tonotopic regions (Fig. 5). After normalization of the peak amplitude, a single exponential was fit to the falling phase of the EPSC for an E11 and E19 neurons recorded from the $\mathrm{HF}$ region of NL (Fig. 5A). The resulting decay tau was 2.5-fold faster for the E19 neuron than the E11 neuron. Similarly, EPSC 10-90\% rise times significantly decreased with age and were quite rapid (Table $1, P<0.0001$ ). The changes in EPSCs kinetics (decay tau and rise times) were similar for recordings using the minimal stimulation protocol (data not shown).

Interestingly, differences in kinetics across the tonotopic region at any given age were less consistent. There were no significant differences in decay tau or rise times between HF and LF neurons at E11 (tau, $P=0.71$; rise, $P=0.14$ ). However, significant differences in decay tau were observed between HF and LF neurons at E13 through E17 $(P<0.05)$. By E19, when responses were the fastest, differences in decay tau across tonotopic regions were less obvious and not statistically significant $(P=0.63)$. However, decay tau for HF neurons was on average faster than LF neurons. Thus neurons from the HF region always exhibited a faster decay tau compared with neurons recorded from the LF region (Fig. 5B). Figure $5 B$ shows example traces recorded from the HF and LF regions in the same NL at E13, E15, and E17. For these neurons, the HF decay tau was $\sim 1.8$-fold (E13), 1.5-fold (E15), and 1.3-fold (E17) faster than the LF decay tau. It should be noted that several factors control the kinetics of AMPA-R mediated EPSCs. These factors include
A

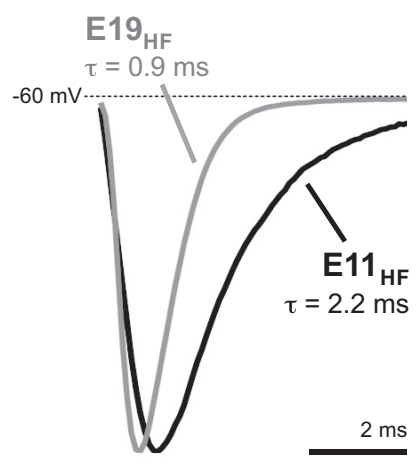

D

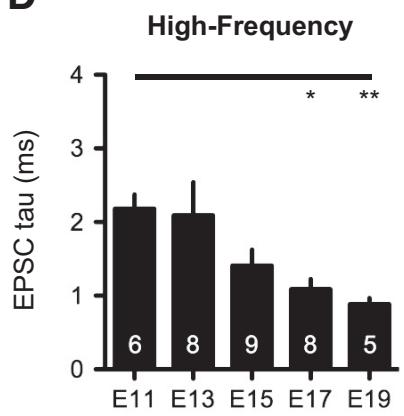

B

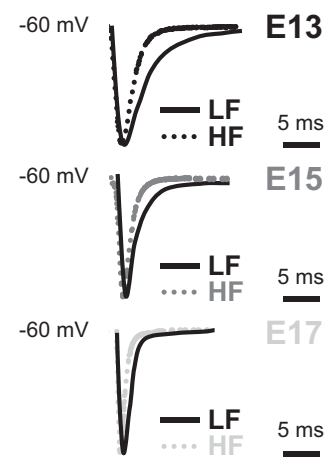

Mid-Frequency

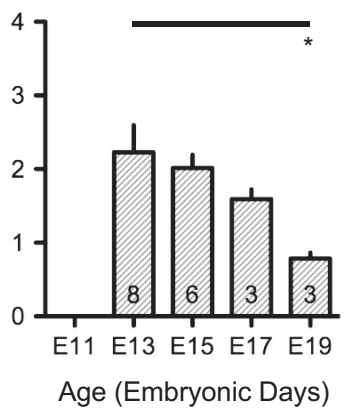

C

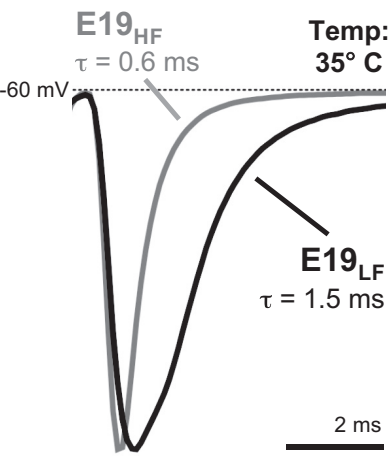

Low-Frequency

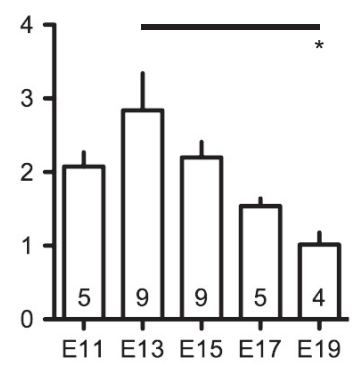

FIG. 5. Isolated AMPA-R currents become faster with age and across tonotopic regions. A: example of normalized AMPA-R mediated EPSCs recorded from the $\mathrm{HF}$ region of $\mathrm{E} 11\left(\mathrm{E} 11_{\mathrm{HF}}\right.$, black trace) and E19 (E19 ${ }_{\mathrm{HF}}$, gray trace) NL neurons. Decay tau $(\tau$, single exponential fit) was $\sim 2.5$-fold faster for the EPSC recorded at E19 vs. the EPSC tau recorded at E11. B: example of normalized AMPA-R mediated EPSCs across tonotopic regions for age-matched neurons. Traces recorded from the same nucleus in the $\mathrm{HF}$ and $\mathrm{LF}$ regions at E13 (HF, black dotted trace; LF, black trace), E15 (HF, gray dotted trace; LF, black trace), and E17 (HF, light gray dotted trace; LF, black trace). $C$ : recording temperature effects decay kinetics. Example of normalized AMPA-R mediated EPSCs recorded from the HF (gray trace) and LF regions (black trace) of an $\mathrm{E} 19 \mathrm{NL}$ neuron at $35^{\circ} \mathrm{C}$. Decay tau was $\sim 2.5$-fold faster for the EPSC recorded from the HF region vs. the EPSC recorded from the LF region. $D$ : population data of decay tau from the $\mathrm{HF}, \mathrm{MF}$, and $\mathrm{LF}$ regions. 
dendritic filtering, glutamate clearance, and receptor subunit composition. The lack of differences in the kinetics of spontaneous mEPSCs across age and tonotopic region (see Fig. 4D) suggests that factors like glutamate clearance (Diamond and Jahr 1997) and dendritic filtering (Magee and Cook 2000; Williams and Mitchell 2008) play a major role only in evoked responses. Previous reports show clear differences in the kinetics of AMPA-R mediated currents between HF and LF neurons (Kuba et al. 2005; Slee et al. 2010). This is likely the result of more physiological temperatures used in the aforementioned studies. We minimized the influence of glutamate dynamics in the synaptic cleft and dendritic filtering by recording EPSCs at room temperature $\left(\sim 21-22^{\circ} \mathrm{C}\right)$ to study subunit composition of iGluRs based on their electrophysiological properties. Control experiments at $35^{\circ} \mathrm{C}$ indeed show larger differences in kinetics across tonotopic regions at older ages (E19; Fig. 5C). Despite the small changes we observe in decay tau, EPSC rise times across tonotopic regions between E11 and E19 were stable and not significantly different (Table 1). Population data are summarized in Fig. $5 D$ showing changes in the evoked AMPA-R mediated EPSC decay tau across age and tonotopic regions in NL. The increase in speed of AMPA-R mediated responses suggests that subunit compo- sition is changing. We next investigated whether the subunit composition of AMPA-Rs changes across age and tonotopic regions in NL.

\section{Increases in AMPA-R mediated inward rectification}

AMPA-Rs containing the GluR2 subunit exhibit slower EPSC kinetics than AMPA-Rs lacking the GluR2 subunit (Lawrence and Trussell 2000; Raman and Trussell 1992; Raman et al. 1994). Also GluR2 lacking AMPA-Rs are permeable to $\mathrm{Ca}^{2+}$ and other divalent ions (Hollmann et al. 1991) and are inwardly rectifying (Williams 1997) due to voltage-dependent blockade by intracellular polyamines (Donevan and Rogawski 1995). We recorded current-voltage $(I-V)$ relationships mediated by AMPA-Rs across age and tonotopic regions to test the hypothesis that the inward rectification and the rapid kinetics of AMPA-R mediated EPSCs observed in older animals are due to a developmental switch in GluR2 content.

We constructed $I-V$ curves with $100 \mu \mathrm{M}$ spermine added to the internal patch pipette solution. Spermine is a polyamine that blocks the ion channel of GluR2-lacking AMPA-Rs in a voltage dependent manner (Bowie and Mayer 1995). Insets in Fig. 6, $A$ and $B$, show recordings of

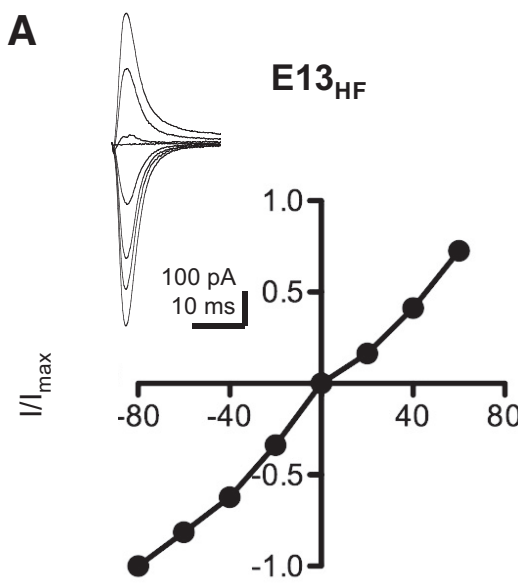

D

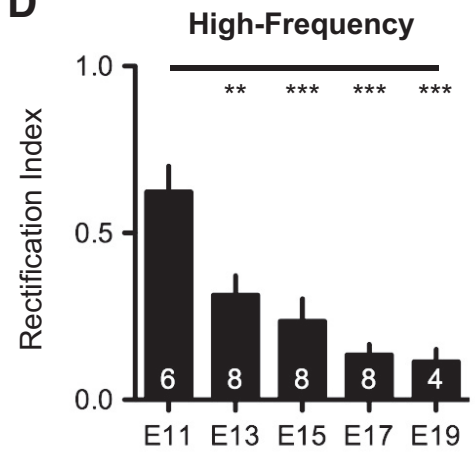

B

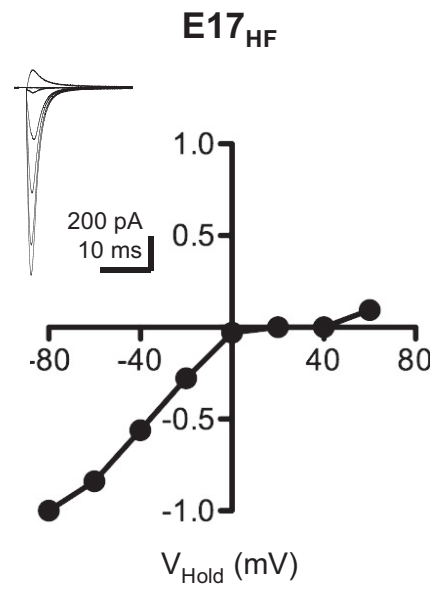

Mid-Frequency

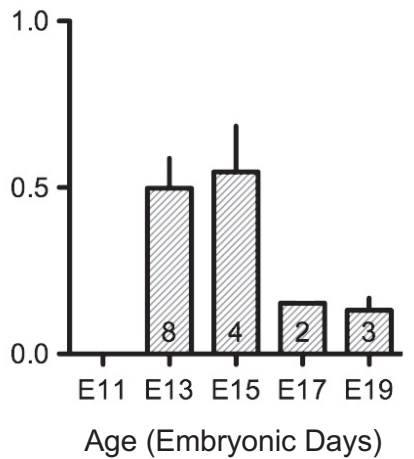

C
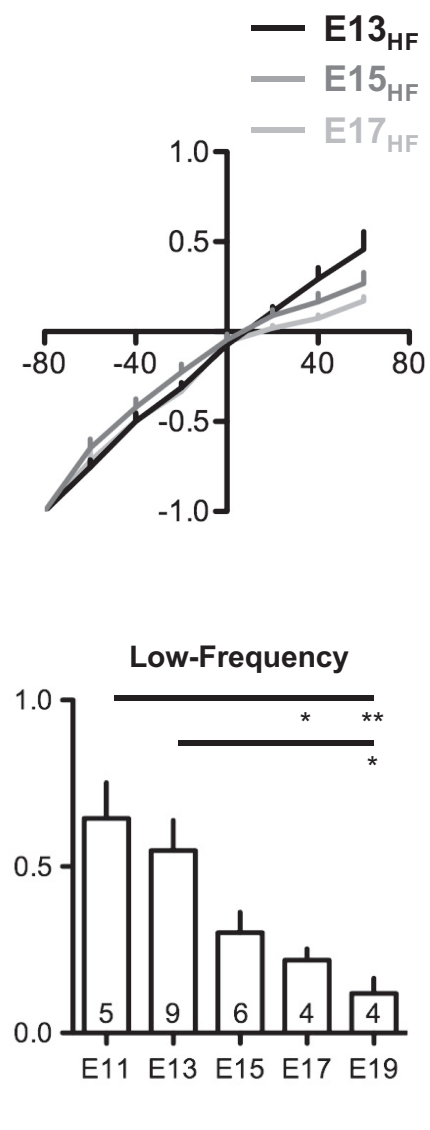

FIG. 6. Increase in rectification of AMPA-R currents with age and across tonotopic regions. $A$ and $B: I-V$ functions recorded from the HF region of E13 ( $A$ ) and E17 (B) NL neurons. Insets: isolated AMPA-R mediated EPSCs recorded at different holding voltages (see METHODS) with $100 \mu \mathrm{M}$ spermine added to pipette internal solution (note different scale bars for insets). $C$ : population $I-V$ functions constructed from NL neurons recorded in the HF region at E13 (HF, black line), E15 (HF, gray line), and E17 (HF, light gray line). $I$ - $V$ plots show a near linear function at E13, changing to an inwardly rectifying $I-V$ function at E17. $D$ : population data of rectification index $\left(I_{\text {AMPA-R }}+40 \mathrm{mV} / I_{\text {AMPA-R }}-60 \mathrm{mV}\right)$ from the HF, MF, and LF regions. 
isolated AMPA-R mediated EPSCs from the HF region of E13 and E17 NL neurons at various holding potentials, from which we constructed normalized $I-V$ curves (Fig. 6, $A$ and $B)$. At E13, the $I-V$ relationship was nearly linear, but at E17, there was a strong reduction in the outward flow of current mediated by isolated AMPA-Rs, consistent with inward rectification. Population $I-V$ functions recorded from the HF region of E13, E15, and E17 NL neurons are overlaid in Fig. $6 C$ and show a near linear function at E13, changing to an inwardly rectifying $I / V$ relationship at E17. The extent of rectification is summarized in Fig. $6 D$ by using a rectification index $\left(\mathrm{RI}=I_{\mathrm{AMPA}-\mathrm{R}}+40 \mathrm{mV} / I_{\mathrm{AMPA}-\mathrm{R}}-60 \mathrm{mV}\right)$ as an indication of AMPA-Rs lacking GluR2 subunit (Bowie and Mayer 1995; Donevan and Rogawski 1995). Population data show a significant increase in AMPA-R mediated rectification with increasing age at every tonotopic region (Fig. $6 D$ ). The rectification index for HF neurons at E11 was 0.62 \pm 0.19 and was $0.11 \pm 0.07$ at E19 $(P<0.0001)$. This profile was similar for MF and LF neurons $(P<0.01$ and $P$ $<0.002$, respectively). These results suggest a dramatic decrease in GluR2-containing AMPA-Rs between E11 and E19. This developmental change in AMPA-R subunit composition could also contribute to the changes in kinetics and amplitude of AMPA-R mediated EPSCs observed during this period of development. However, the lack of consistent differences across tonotopic regions at a given age suggests that other mechanisms could contribute to the differences observed in AMPA-R mediated EPSCs kinetics as well.

\section{Kainate-induced cobalt $\left(\mathrm{Co}^{2+}\right)$ uptake reveals GluR2-lacking AMPA-Rs}

To further investigate changes in GluR2-receptor subunit expression in developing NL neurons, we used $\mathrm{Co}^{2+}$ uptake to label neurons with GluR2-lacking AMPA-Rs (Estabel et al. 1999; Pruss et al. 1991; Zhou et al. 1995). We show that kainate-induced $\mathrm{Co}^{2+}$ uptake is robust in NL, suggesting $\mathrm{Ca}^{2+}$-permeable GluR2-lacking AMPA-Rs (Fig. 7, $C$ and $D$ ). For control experiments, we exposed slices to $\mathrm{CoCl}_{2}$ only or $\mathrm{CoCl}_{2}$ plus kainate and DNQX, an AMPA-R agonist and antagonist, respectively. Nomarski-DIC photomicrographs illustrate the absence of $\mathrm{Co}^{2+}$ uptake in NL neurons under these control conditions (Fig. 7, $A$ and $B$ ). Figure 7, $C$ and $D$, shows kainate-induced $\mathrm{Co}^{2+}$ uptake in slices taken from the HF region of E11 and E19, respectively. Incubation with kainate for 20 min was sufficient to produce strong $\mathrm{Co}^{2+}$ labeling. NL cell bodies contained robust staining, while the $\mathrm{Co}^{2+}$ uptake in both dorsal and ventral neuropil regions was less concentrated. $\mathrm{Co}^{2+}$ uptake was similar at E15, E21, and P4 slices (data not shown). This strong labeling was completely blocked by the AMPA-R antagonist DNQX (data not shown), suggesting that $\mathrm{Co}^{2+}$ is indeed entering through AMPA-Rs. Presence of $\mathrm{Co}^{2+}$
A

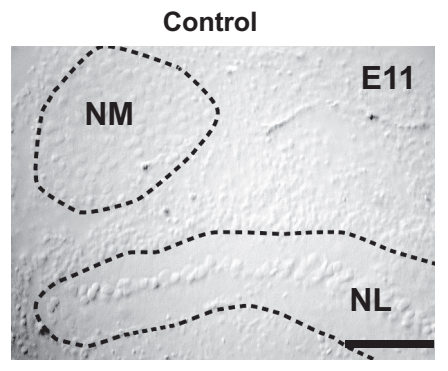

B

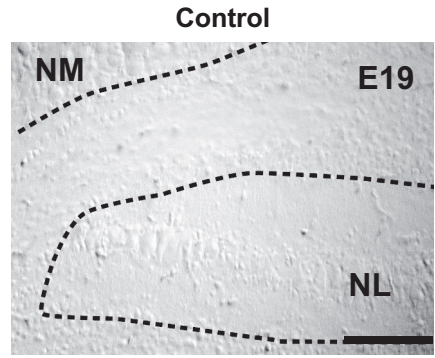

C

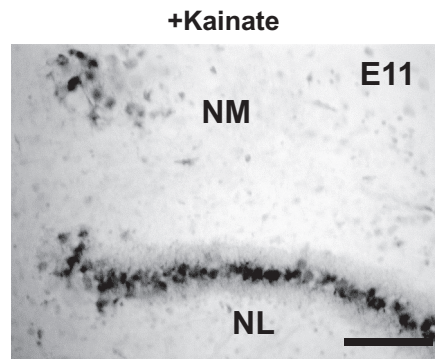

D

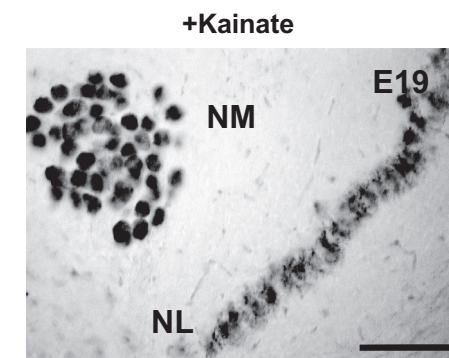

$\mathbf{E}$

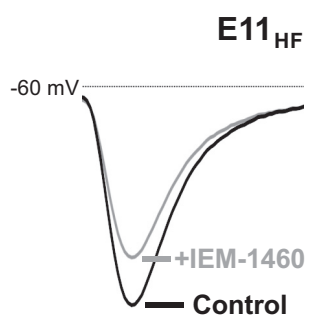

$\mathbf{F}$

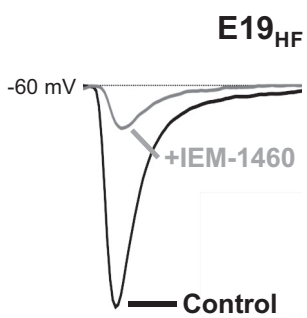

$50 \mathrm{pA}$

$1 \mathrm{~ms}$
$250 \mathrm{pA}$

$1 \mathrm{~ms}$
G

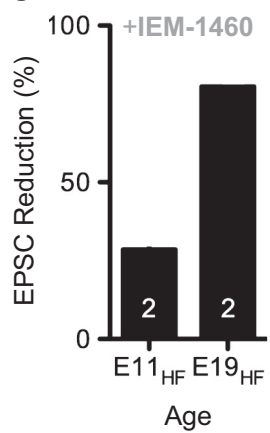

FIG. 7. Kainate-induced $\mathrm{Co}^{2+}$ uptake suggests GluR2-lacking AMPA-Rs. $A$ and $B$ : Nomarski-DIC photomicrographs illustrating the absence of $\mathrm{Co}^{2+}$ uptake in slices incubated in ACSF containing $5 \mathrm{mM} \mathrm{CoCl}_{2}$ only (control) for E11 $(A)$ and E19 (B) tissue. Dashed lines indicate the location of NM and NL. Scale bars $=100 \mu \mathrm{m}$. $C$ and $D$ : bright field photomicrographs illustrating kainate-induced $\mathrm{Co}^{2+}$ uptake in E11 (C) and E19 (D) slices. NL cell bodies contain robust staining, while the $\mathrm{Co}^{2+}$ uptake in both neuropil regions is less concentrated. Scale bars $=100 \mu \mathrm{m}$. Kainateinduced $\mathrm{Co}^{2+}$ was blocked at all ages tested with bath application of 6,7-dinitroquinoxaline-2,3(1H,4H)-dione (DNQX, $50 \mu \mathrm{M}$, data not shown). $E$ and $F$ : effect of specific GluR2-lacking AMPA-R antagonist. Example traces of AMPA-R mediated EPSCs before (control, black traces) and after (gray traces) bath application of $N, N, N$,-rimethyl-5-[(tricyclo[3.3.1.13,7]dec-1-ylmethyl )amino]-1pentanaminiumbromide hydrobromide (IEM-1460, $100 \mu \mathrm{M}$ ) from E11 $(E)$ and E19 $(F)$ slices recorded from the HF region. Note different scale bars. $G$ : summary data showing percent reduction by IEM-1460 at E11 and E19. 
uptake throughout NL suggests that neurons express $\mathrm{Ca}^{2+}$ permeable GluR2-lacking AMPA-Rs early and late in development and across different tonotopic regions of NL. A gradient in $\mathrm{Co}^{2+}$ uptake across different tonotopic regions was not consistently observed, which may be due to inadequate sensitivity of the method (see next section).

The strong $\mathrm{Co}^{2+}$ uptake at E11 was somewhat surprising based on the electrophysiology data. The EPSC decay tau was relatively slow for E11 neurons recorded from the HF regions $(\operatorname{tau}=2.17 \pm 0.47, n=6$, see Fig. 5), and the rectification index showed near linearity $(\mathrm{RI}=0.62 \pm 0.19, n=6$, see Fig. 6 ), both of which are considered reliable biophysical markers that indicate GluR2-containing AMPA-Rs. However, it should be noted that exposure to $\mathrm{CoCl}_{2}$ and kainate for time periods $<10$ min produced results with highly variable staining patterns, whereas treatments $>15$ min always resulted in robust staining consistent with a saturation effect. An interpretation of these data, consistent with other developmental research (Pellegrini-Giampietro et al. 1997), is that NL neurons contain both GluR2-containing and -lacking AMPA-Rs early in development and exclusively or primarily GluR2-lacking AMPA-Rs by E17-19 and that differential expression of each type is indistinguishable with the $\mathrm{Co}^{2+}$-uptake technique.

To better resolve this issue, we bath applied a dicationic adamantine derivative (IEM-1460, $100 \mu \mathrm{M}$ ), a highly specific antagonist to the GluR2-lacking AMPA-R, to determine the relative contribution of this $\mathrm{Ca}^{2+}$ permeant AMPA-R to the overall current response when NL neurons were voltageclamped at $-60 \mathrm{mV}$. Representative traces recorded from the HF region of E11 and E19 NL neurons are shown in Fig. 7, $E$ and $F$, respectively. For the E11 neuron, the control EPSC using the maximum stimulus protocol was $-350 \mathrm{pA}$ but was reduced to $-275 \mathrm{pA}$ (21\% reduction) when IEM-1460 was bath applied for 15 min (Fig. 7E). On average, the EPSC decay tau was slightly faster in the control responses than after treatment with the drug (control $_{\text {tau }}=2.14 \pm 0.41 \mathrm{~ms} ;$ drug $\left._{\text {tau }}=2.37 \pm 0.57 \mathrm{~ms}\right)$. These data indicate a small contribution of GluR2 lacking AMPA-Rs to the total AMPA-R mediated EPSC at E11. Thus the kainate induced $\mathrm{Co}^{2+}$ uptake observed at E11 is likely due to the contribution of a small percentage of GluR2 lacking AMPA-Rs. In contrast, at E19, the control maximum EPSC was $-1,700 \mathrm{pA}$ but was reduced considerably to $-320 \mathrm{pA}$ ( $81 \%$ reduction) when the GluR2-lacking AMPA-Rs were blocked (Fig. 7F). On average, the EPSC decay time constant of the control responses was considerably faster than after application of the drug $\left(\right.$ control $_{\text {tau }}=$ $0.88 \pm 0.05$; drug $_{\text {tau }}=1.07 \pm 0.16$ ). These data indicate that the majority of the current at E19 is mediated by AMPA-Rs lacking the GluR2 subunit. Population data showing the percent of EPSC reduction following blockade of GluR2-lacking AMPA-Rs are summarized in Fig. $7 G$.

\section{Changes in the amplitude of evoked NMDA-R mediated EPSCs}

Pharmacologically isolated NMDA-R mediated EPSCs were recorded while holding the membrane potential at $+40 \mathrm{mV}$. A maximal stimulation protocol was used to examine changes in the amplitude of evoked EPSCs with development and across different tonotopic regions. I/O functions were determined for each NL neurons in an attempt to recruit most, if not all, excitatory inputs from the ipsilateral NM. The stimulus intensity used to evoke maximum NMDA-R currents was determined from individual $\mathrm{I} / \mathrm{O}$ functions and an example is shown in Fig. 8A. NMDA-R currents increased with increases in stimulus intensity with few failures at minimal stimulation consistent with a high probability of release. The stimulus intensity used to determine a maximum response was chosen when the evoked EPSC reached a plateau response (arrow in Fig. 8A). This stimulus intensity never exceeded the output of the stimulus generator.

NL neurons showed dramatic changes with age in the amplitude of maximally evoked NMDA-R mediated EPSCs. Representative traces from the HF region of E13, E15, E17, and E19 NL neurons are shown in Fig. 8B. For HF neurons,
A

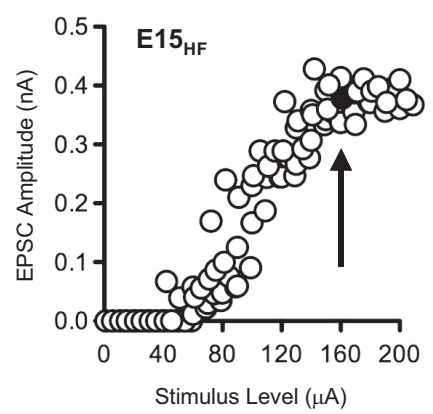

B

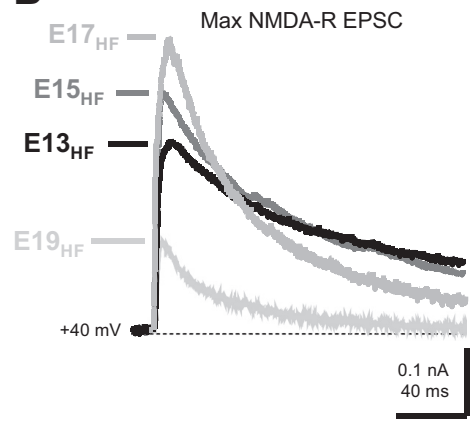

C

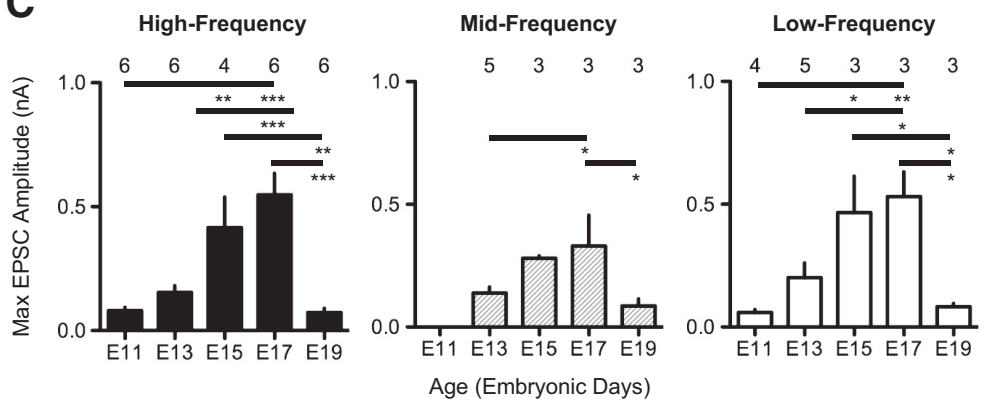

FIG. 8. Changes in isolated NMDA-R currents during development and across tonotopic regions. $A$ : isolated NMDA-R mediated EPSCs plotted as a function of stimulus intensity (average of 5 trials; open circles) recorded from the HF region of an E15 NL neuron (same cell in $B$ ). Arrow indicates stimulus intensity used to determine maximum NMDA-R current (filled circle). $B$ : maximum NMDA-R mediated EPSC peak amplitudes changes with age. Representative recordings from the HF regions of E13 (black trace) E15 (dark gray trace), E17 (gray trace), and E19 (light gray trace) NL neurons. Responses were recorded in the presence of $20 \mu \mathrm{M}$ NBQX while holding the membrane potential at $+40 \mathrm{mV}$. Dash line represents the baseline. $C$ : population data of maximum NMDA-R mediated EPSCs from the HF, MF, and LF regions. 
NMDA-R responses increased approximately sevenfold from E11 to E17, followed by a sharp and significant decline in amplitude at E19 (Fig. 8, $B$ and $C$ ) to values similar to those observed at E11 (Ell trace not shown in $B$ for clarity). In fact, the average NMDA-R currents recorded at E11 and E19 were not significantly different from one another $(P=0.72)$. This profile was similar in each tonotopic region of NL. MF neurons showed a modest, yet significant 2.5-fold increase from E13 to E17 (Fig. 8C, middle) and LF neurons resulted in a ninefold increase from E11 to $\mathrm{E} 17$ (C, right). These results were also observed using a minimal stimulation protocol of afferent inputs from $\mathrm{NM}$ as determined by individual $\mathrm{I} / \mathrm{O}$ functions (data not shown). No significant differences in the maximum amplitude were observed across different tonotopic regions at any particular age regardless of the difference in dendritic elaboration, supporting the idea that space clamp issues across different tonotopic regions did not affect the amplitude of EPSCs.

\section{Changes in NMDA-R mediated EPSC kinetics}

NMDA-R subunit composition defines the kinetics of evoked EPSCs (Dingledine et al. 1999). Particularly important are NR2A and NR2B subunits, the expression of which is temporally and spatially regulated in a variety of neuronal systems (Dingledine et al. 1999; Sabatini and Regehr 1999; Wu et al. 1999). We observed acceleration in the NMDA-R mediated EPSC decay tau throughout development, indicating a switch in NR2 subunit composition from NR2B to NR2A. An example is shown in Fig. 9A. After normalization of the peak amplitude, a weighted exponential (see METHODs) was fit to the falling phase of the EPSC for an E11 and an E19 neuron recorded from the HF region of NL. The resulting decay tau was 4.5 -fold faster for the E19 neuron.

To better assess a development switch in NMDA-R subunits, we recorded from the HF region of E11 and E19 neurons in the presence of a specific NR2B subunit antagonist (Ro256981, $1 \mu \mathrm{M}$ ) (Fischer et al. 1997; Kosowski and Liljequist 2004; Lynch et al. 2001) to determine the relative contribution of the NR2B subunit to the overall NMDA-R current response when NL neurons were voltage-clamped at $+40 \mathrm{mV}$. Representative traces are shown in Fig. 9B. For the E11 neuron (top), the control EPSC using the maximum stimulus protocol was $148 \mathrm{pA}$ and was significantly reduced to $39 \mathrm{pA}$ (74\% reduction) when the NR2B antagonist Ro25-6981 was bath applied for $16 \mathrm{~min}$ (Fig. 9C). On average, the EPSC decay time constant was slightly faster in the drug condition than in the control condition (control $_{\mathrm{tau}}=143.20 \pm 17.31 \mathrm{~ms}$; drug $_{\mathrm{tau}}=$ $99.50 \pm 29.57 \mathrm{~ms}$ ). These data indicate a major contribution of
A

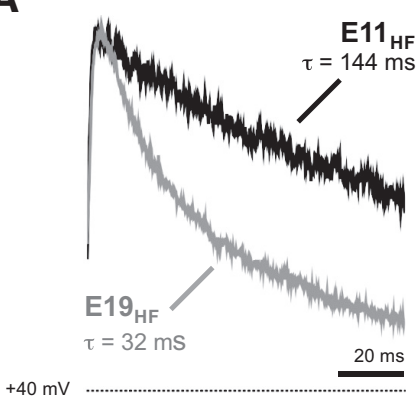

C

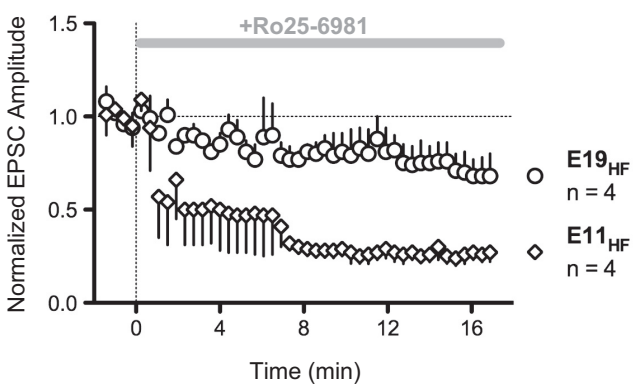

$\mathbf{E}$

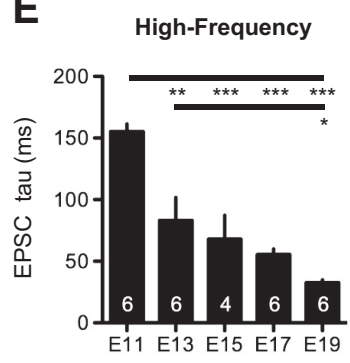

B

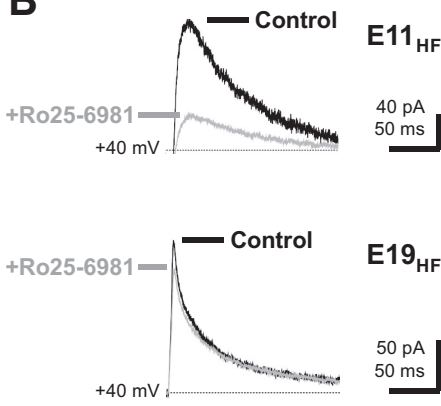

D

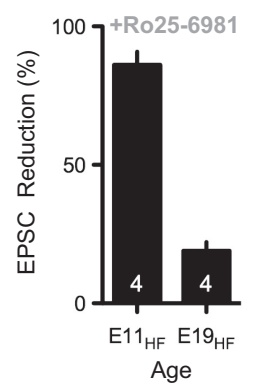

Low-Frequency

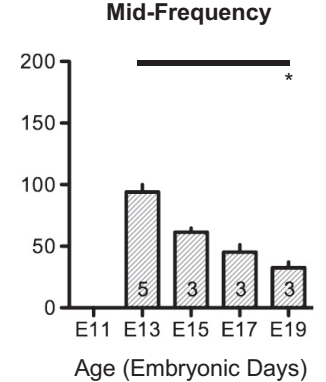

FIG. 9. Developmental changes in NMDA-R kinetics reflect a switch in subunit composition. $A$ : normalized NMDA-R mediated EPSCs recorded from the HF region of E11 (E11 $1_{\mathrm{HF}}$, black trace) and E19 $\left(\right.$ E19 ${ }_{\mathrm{HF}}$, gray trace) NL neurons. Decay constant $(\tau$, weighted exponential fit, see METHODS) was $\sim 4.5$-fold faster for the EPSC recorded at E19 compared with E11. Traces were recorded in the presence of $20 \mu \mathrm{M}$ NBQX while holding the membrane potential at +40 mV. Dashed lines represent baseline. $B$ : representative traces recorded from the HF region of E11 (top) and E19 (bottom) NL neurons before (control, black traces) and after specific blockade of the NR2B-containing NMDA-Rs [+(aR,bS)-a-(4-hydroxyphenyl)b-methyl-4-(phenylmethyl)-1-piperidinepropanol maleate (+Ro256981), $1 \mu \mathrm{M}$, gray traces]. $C$ : population data showing the time course of NR2B blockade with Ro25-6981 for E11 (open diamond) and E19 (open circle) HF neurons. D: summary data showing percent reduction of Ro25-6981 at E11 and E19. E: population data of decay tau from the HF, MF, and LF regions. 
the NR2B subunit to the NMDA-R mediated EPSC at E11. In contrast, at E19, the control maximum EPSC was 145 pA but was minimally reduced to $118 \mathrm{pA}$ ( $\sim 19 \%$ reduction) when NR2B-containing NMDA-Rs were blocked for 16 min (Fig. 9, $B$, bottom, and $C$ ). On average, the EPSC decay tau of the control responses was not different after application of the drug $\left(\right.$ control $_{\text {tau }}=39.17 \pm 6.39 ;$ drug $\left._{\text {tau }}=34.16 \pm 4.79\right)$, indicating that the majority of the current at E19 is mediated by NMDA-Rs lacking the NR2B subunit. Population data showing the percent of EPSC reduction following blockade of NR2B-containing NMDA-Rs are summarized in Fig. 9D.

This acceleration of NMDA-R responses was similar and significant across age for all tonotopic regions, with HF neurons resulting in a 4.7-fold increase (Fig. 9E, left), MF neurons increasing threefold from E13 to E19 (Fig. 9E, middle), and LF neurons increasing 3.1-fold from E11 to E19 (Fig. 9E, right). The acceleration in the kinetics of NMDA-R dependent EPSCs was similar for recordings using the minimal stimulation protocol (data not shown). In contrast, differences in kinetics across tonotopic region for age-matched neurons were not apparent, suggesting that unlike AMPA-R development, NMDA-Rs are not differentially regulated tonotopically. These results further argue against space-clamp issues associated with dendritic filtering. Population data summarized in Fig. 9E, along with specific blockade of the NR2B subunit summarized in $B-D$, strongly suggest a developmental switch in NMDA-R subunit composition from NR2B- to NR2A-containing receptors in NL neurons during this period of development.

\section{I S C U S S I ON}

In several brain regions, synaptic expression of AMPA-Rs and NMDA-Rs change throughout development, fine-tuning synaptic properties, and facilitating proper dendritic and neuronal circuit maturation (Cline and Haas 2008; Haas et al. 2006). Synaptic maturation increases the AMPA/NMDA ratio (Hall and Ghosh 2008; Kasanetz and Manzoni 2009; Rumpel et al. 2004) and changes the subunit composition of NMDA-Rs, altering integration of presynaptic inputs, $\mathrm{Ca}^{2+}$ influx into the postsynaptic neuron, and the ability of the synapse to undergo plasticity (Barria and Malinow 2005; Carmignoto and Vicini 1992; Crair and Malenka 1995; Hestrin 1992; Lu et al. 2001; Philpot et al. 2001).

In NL, the first neuronal circuit that integrates binaural information from both ears in the avian brain stem, AMPA-R currents are critical in shaping extremely rapid EPSPs, a physiologic necessity for binaural auditory processing (Trussell 1997). The role of NMDA-Rs is less clear. It has been suggested that responses mediated by NMDA-Rs disappear shortly after synaptogenesis, contributing to the sharpening of EPSPs (Gao and $\mathrm{Lu}$ 2008; Kuba et al. 2002a). However, robust expression levels of NMDA-Rs are evident into adulthood (Tang and Carr 2004, 2007).

Our results indicate that NL synapses undergo major postsynaptic changes in the strength and subunit composition of AMPA-Rs and NMDA-Rs prior to hatching. These dramatic changes correlate to a developmental time period when the characteristic morphology of NL neurons and circuitry are being established, the tonotopic gradient is being defined, and hearing is emerging. These changes seem to occur initially in the $\mathrm{HF}$ region of NL, a pattern that mimics receptor develop- ment and other morphological properties in NM and NL, suggesting that the development of glutamatergic synaptic transmission is regulated by the activity of their sensory inputs. These developmental changes increase the amplitude and speed of glutamatergic synaptic transmission, likely enhancing coincidence detection of binaural auditory information.

\section{Development of synaptic AMPA-Rs in NL}

We observe significant age and tonotopic dependence in the amplitude and kinetics of EPSCs mediated by AMPA-Rs in NL. These results reflect increases in synaptic contacts and changes in receptor subunit composition. Similar changes as a function of age have been reported in different relay synapses such as the calyx of Held synapse in the mouse (Joshi and Wang 2002), the endbulb of Held synapse in the rat (Bellingham et al. 1998), and the endbulb synapse of the chick NM (Lu and Trussell 2007).

The increase in AMPA- $R$ responses can be attributed to either an upregulation of presynaptic voltage-gated $\mathrm{Ca}^{2+}$ channels resulting in an increase in release probability (Brenowitz and Trussell 2001; Kasanetz and Manzoni 2009), an increase in the number of postsynaptic AMPA-Rs (Elias et al. 2006, 2008; Turrigiano 2008), or an increase in the number of synaptic contacts innervating the postsynaptic neuron (Brewer et al. 2009; Hackett et al. 1982). We observe no changes throughout development in the amplitude of mEPSCs and no changes in the probability of release. In addition, the stepwise increase of AMPA-R mediated responses observed in the input/output curves and the lack of differences in the rise time of EPSCs across different ages suggest no differences in the synchronization of transmitter release during development. Thus it is unlikely that presynaptic changes account for the large increase in AMPA-R mediated transmission observed. The increase in mEPSCs frequency suggests that the increase in the amplitude of AMPA-R responses is due to an increase in the number of synaptic contacts (Hsia et al. 1998; Petralia et al. 1999), although it does not rule out changes in receptor density. Interestingly, the increase in AMPA-R responses is first apparent in the HF region, conforming to the gradient in synaptic development first reported by Jackson et al. (1982) and similar to NM development (Lu and Trussell 2007). Neurons located in the HF region of NL had larger amplitude EPSCs compared with the MF and LF regions, suggesting that afferent inputs from NM make more synaptic contacts with HF neurons despite considerable size differences in dendritic arborization compared with LF neurons (Smith and Rubel 1979) (see also Fig. 1). We cannot rule out the contribution of differential filtering due to dendritic length on the peak amplitude of AMPA-R mediated EPSCs; however, we did not observe tonotopic changes in the peak amplitude of NMDA-R mediated responses (discussed in the next section). Thus our results indicate that the increase in AMPA-R mediated responses is due primarily to increases in synaptic contacts. We also report that AMPA-Rs in NL undergo a switch in subunit composition, from a predominantly small conductance channel at E11 (i.e., containing the GluR2 receptor subunit), to a large conductance channel at E19 (i.e., lacking the GluR2 receptor subunit), which may further contribute to an increase in the amplitude of AMPA-R currents (Conrad et al. 2008; Cull-Candy et al. 2006; Dingledine et al. 1999). 
Inward rectification, $\mathrm{Co}^{2+}$ uptake, and pharmacological blockade indicate a small proportion of GluR2-lacking AMPA-Rs at E11 that increases dramatically with age. However, unlike increases in peak amplitude of AMPA-R mediated EPSC, inward rectification did not appear to emerge differentially across the tonotopic gradient. It should also be noted that our finding of late tissue ( $>\mathrm{E} 19) \mathrm{Co}^{2+}$ uptake is in contrast to those reported by Zhou et al. (1995). We suggest that the failure to observe $\mathrm{Co}^{2+}$ uptake in their study of late embryos was due to the lack of adequate tissue penetration in the intact brain stem preparation.

We also observe acceleration in the decay kinetics of AMPA-R responses with age. Channel gating, desensitization, subunit composition, dynamics of glutamate in the synaptic cleft, and passive properties of dendrites can influence the decay kinetics of AMPA-R mediated EPSCs (Parks 2000; Trussell 1998). We recorded EPSCs at room temperature to minimize dendritic filtering and the influence of glutamate dynamics in the synaptic cleft, two factors highly dependent on temperature (Tzingounis and Wadiche 2007). Our data suggest that the subunit composition changes in a direction that increases the kinetics of AMPA-R responses (i.e., from predominately GluR2 containing to GluR2-lacking receptors). Therefore it is reasonable to expect that the stabilization of response kinetics we observe occur because the change in AMPA-R subunit composition is near complete at older ages. Our data at E19 show minimal differences in response kinetics between HF and LF neurons, suggesting maximal replacement of AMPA-R subunits across the tonotopic gradient prior to hatching. Consequently, if the composition of receptors becomes homogenous across tonotopic regions, factors like glutamate clearance and dendritic filtering become dominant in defining the kinetics of EPSCs. It is clear from previous studies in older tissue and at more physiological temperatures, that dendritic filtering plays a major role in defining tonotopic differences in kinetics of AMPA-R mediated EPSCs (Kuba et al. 2005; Slee et al. 2010).

NL neurons have both intrinsic and synaptic properties that are extremely brief to accommodate rapid transmission (Parks 2000; Trussell 1997, 1999). We propose that a developmental increase in GluR2-lacking AMPA-Rs helps sharpen the EPSP time course, thus minimizing distortion, a requirement for binaural auditory processing (Otis et al. 1995; Raman et al. 1994). Additionally, an increase in $\mathrm{Ca}^{2+}$ permeability, due to GluR2-lacking AMPA-R receptors, could be necessary for dendritic remodeling and expression of synaptically regulated extrusion mechanisms (Wang and Rubel 2008). However, $\mathrm{Ca}^{2+}$-permeable AMPA-Rs could be a consequence of the auditory system requiring fast AMPA-Rs (Parks 2000; Trussell 1999), and the reported $\mathrm{Ca}^{2+}$ influx through these receptors is relatively low (Otis et al. 1995). Further work needs to be performed to elucidate the functional role of increased $\mathrm{Ca}^{2+}$ permeability due to GluR2-lacking AMPA-Rs in the auditory system.

\section{Development of synaptic NMDA-Rs in NL}

As soon as synapses are established in NL $(\sim$ E10), current responses are mediated by both AMPA-Rs and NMDA-Rs with a ratio close to one. Due to the large increase in AMPA-R transmission, it is easy to underestimate the contribution of
NMDA-Rs in later stages of development (Funabiki et al. 1998; Zhou and Parks 1991). Similarly to AMPA-Rs, we observe an initial increase in synaptic NMDA-R responses, which peak at E17, followed by a dramatic and significant decrease by E19 to values similar to E11. Subunit composition of NMDA-Rs alters the kinetics of NMDA-R mediated EPSCs (Dingledine et al. 1999). We observe dramatic changes in isolated NMDA-R decay kinetics from $\sim 150 \mathrm{~ms}$ decay time constant at E11 to $\sim 30 \mathrm{~ms}$ at E19. These results, along with our specific blockade of NR2B-containing receptors, indicate a switch in subunit composition from NR2B-containing receptors early in synaptic development to NR2A-containing receptors later in development, as shown for other developing central synapses (Carmignoto and Vicini 1992; Flint et al. 1997; Tovar and Westbrook 1999). This developmental switch in subunit composition accompanies significant changes in channel kinetics, which plays key roles in regulating $\mathrm{Ca}^{2+}$ influx, organizing functional circuits, regulating dendritic growth, and refining topographic projections (Cline 2001; Ruthazer and Cline 2004; Sin et al. 2002).

This pattern of development for NMDA-Rs is different from NM neurons where no developmental change in NMDA-R subunit composition is observed and synaptic NMDA-R responses disappear by E19 (Lu and Trussell 2007). This difference in the development of NMDA-Rs highlights the diverse roles of each synapse in the transmission and processing of auditory information. It also suggests that NMDA-Rs could play a role in the precise timing and processing of binaural information by contributing to firing probability, response latency, and spike jitter (Pliss et al. 2009; Sanchez et al. 2007). In addition, NMDA-Rs could be critical for synaptic stability, dendritic maintenance, and circuit refinement (Gillespie et al. 2005).

\section{Functional role of NMDA-Rs in NL}

Little is known about the functional role of NMDA-Rs in NL. Both light and electron microscopy studies in NL have shown the presence of NMDA-R subunits during development and into adulthood in a pattern of expression consistent with our functional studies (Tang and Carr 2004, 2007). In view of the critical role of NMDA-Rs in development, and plasticity of many immature synapses (Rajan and Cline 1998; Rajan et al. 1999), it is tempting to speculate that the transient but dramatic increase of NMDA-R function during synaptic formation in NL may provide the appropriate $\mathrm{Ca}^{2+}$ signaling for incorporation, stabilization, and subunit re-arrangement of AMPA-Rs in the postsynaptic density and/or other specializations such as the emergence of channel specializations important for binaural processing and the dendritic gradient. Downregulation of synaptic NMDA-Rs could stabilize the system as well as prevent $\mathrm{Ca}^{2+}$ overloading due to high rates of spontaneous and acoustically driven activity (Born et al. 1991).

The current study describes dramatic changes in glutamatergic synaptic transmission in NL during embryonic development and provides a unique platform to evaluate relationships between the developmental expression of synaptic receptors and other structure/function specializations of a well-defined neuronal circuit. 


\section{A C K N O W LED G M EN TS}

We thank current members of the Rubel and Barria labs for helpful discussion of the data and manuscript. We also thank the critiques of three reviewers for improving the manuscript.

\section{G R A N T S}

This work was supported by National Institute on Deafness and Other Communications Disorders Research Grant R01 DC-003829 and National Research Service Award F32 DC-010307.

\section{I S C L O S URES}

No conflicts of interest, financial or otherwise, are declared by the author(s).

\section{R EFEREN C ES}

Barria A, Malinow R. NMDA receptor subunit composition controls synaptic plasticity by regulating binding to CaMKII. Neuron 48: 289-301, 2005.

Bellingham MC, Lim R, Walmsley B. Developmental changes in EPSC quantal size and quantal content at a central glutamatergic synapse in rat. $J$ Physiol 511: 861-869, 1998.

Born DE, Durham D, Rubel EW. Afferent influences on brainstem auditory nuclei of the chick: nucleus magnocellularis neuronal activity following cochlea removal. Brain Res 557: 37-47, 1991.

Bowie D, Mayer ML. Inward rectification of both AMPA and kainate subtype glutamate receptors generated by polyamine-mediated ion channel block. Neuron 15: 453-462, 1995.

Brenowitz S, Trussell LO. Minimizing synaptic depression by control of release probability. J Neurosci 21: 1857-1867, 2001.

Brewer GJ, Boehler MD, Pearson RA, DeMaris AA, Ide AN, Wheeler BC. Neuron network activity scales exponentially with synapse density. J Neural Eng 6: 1-12, 2009.

Carmignoto G, Vicini S. Activity-dependent decrease in NMDA receptor responses during development of the visual cortex. Science 258: 1007-1011, 1992.

Cline HT. Dendritic arbor development and synaptogenesis. Curr Opin Neurobiol 11: 118-126, 2001.

Cline H, Haas K. The regulation of dendritic arbor development and plasticity by glutamatergic synaptic input: a review of the synaptotrophic hypothesis. J Physiol 586: 1509-1517, 2008.

Conrad KL, Tseng KY, Uejima JL, Reimers JM, Heng LJ, Shaham Y, Marinelli M, Wolf ME. Formation of accumbens GluR2-lacking AMPA receptors mediates incubation of cocaine craving. Nature 454: 118-121, 2008.

Crair MC, Malenka RC. A critical period for long-term potentiation at thalamocortical synapses. Nature 375: 325-328, 1995.

Cull-Candy S, Kelly L, Farrant M. Regulation of $\mathrm{Ca}^{2+}$-permeable AMPA receptors: synaptic plasticity and beyond. Curr Opin Neurobiol 16: 288 297, 2006.

Diamond JS, Jahr CE. Transporters buffer synaptically released glutamate on a submillisecond time scale. J Neurosci 17: 4672-4687, 1997.

Diaz C, Martinez-Galan JR, Juiz JM. Development of glutamate receptors in auditory neurons from long-term organotypic cultures of the embryonic chick hindbrain. Eur J Neurosci 29: 213-230, 2009.

Dingledine R, Borges K, Bowie D, Traynelis SF. The glutamate receptor ion channels. Pharmacol Rev 51: 7-61, 1999.

Donevan SD, Rogawski MA. Intracellular polyamines mediate inward rectification of $\mathrm{Ca}(2+)$-permeable alpha-amino-3-hydroxy-5-methyl-4-isoxazolepropionic acid receptors. Proc Natl Acad Sci USA 92: 9298-9302, 1995.

Durand GM, Kovalchuk Y, Konnerth A. Long-term potentiation and functional synapse induction in developing hippocampus. Nature 381: 71-75, 1996.

Elias GM, Elias LA, Apostolides PF, Kriegstein AR, Nicoll RA. Differential trafficking of AMPA and NMDA receptors by SAP102 and PSD-95 underlies synapse development. Proc Natl Acad Sci USA 105: 20953-20958, 2008.

Elias GM, Funke L, Stein V, Grant SG, Bredt DS, Nicoll RA. Synapsespecific and developmentally regulated targeting of AMPA receptors by a family of MAGUK scaffolding proteins. Neuron 52: 307-320, 2006.

Espinosa JS, Wheeler DG, Tsien RW, Luo L. Uncoupling dendrite growth and patterning: single-cell knockout analysis of NMDA receptor 2B. Neuron 62: 205-217, 2009.
Estabel J, Konig N, Exbrayat JM. AMPA/kainate receptors permeable to divalent cations in amphibian central nervous system. Life Sci 64: 607-616, 1999.

Esteban JA, Shi SH, Wilson C, Nuriya M, Huganir RL, Malinow R. PKA phosphorylation of AMPA receptor subunits controls synaptic trafficking underlying plasticity. Nat Neurosci 6: 136-143, 2003.

Fischer G, Mutel V, Trube G, Malherbe P, Kew JN, Mohacsi E, Heitz MP, Kemp JA. Ro 25-6981, a highly potent and selective blocker of N-methyld-aspartate receptors containing the NR2B subunit. Characterization in vitro. J Pharmacol Exp Ther 283: 1285-1292, 1997.

Flint AC, Maisch US, Weishaupt JH, Kriegstein AR, Monyer H. NR2A subunit expression shortens NMDA receptor synaptic currents in developing neocortex. J Neurosci 17: 2469-2476, 1997.

Funabiki K, Koyano K, Ohmori H. The role of GABAergic inputs for coincidence detection in the neurons of nucleus laminaris of the chick. $J$ Physiol 508: 851-869, 1998.

Gao H, Lu Y. Early development of intrinsic and synaptic properties of chicken nucleus laminaris neurons. Neuroscience 153: 131-143, 2008.

Gillespie DC, Kim G, Kandler K. Inhibitory synapses in the developing auditory system are glutamatergic. Nat Neurosci 8: 332-338, 2005.

Haas K, Li J, Cline HT. AMPA receptors regulate experience-dependent dendritic arbor growth in vivo. Proc Natl Acad Sci USA 103: 12127-12131, 2006.

Hackett JT, Jackson H, Rubel EW. Synaptic excitation of the second and third order auditory neurons in the avian brain stem. Neuroscience 7: 1455-1469, 1982.

Hall BJ, Ghosh A. Regulation of AMPA receptor recruitment at developing synapses. Trends Neurosci 31: 82-89, 2008.

Hestrin S. Developmental regulation of NMDA receptor-mediated synaptic currents at a central synapse. Nature 357: 686-689, 1992.

Hollmann M, Hartley M, Heinemann S. $\mathrm{Ca}^{2+}$ permeability of KA-AMPAgated glutamate receptor channels depends on subunit composition. Science 252: 851-853, 1991.

Howard MA, Burger RM, Rubel EW. A developmental switch to GABAergic inhibition dependent on increases in Kv1-type $\mathrm{K}^{+}$currents. J Neurosci 27: 2112-2123, 2007.

Hsia AY, Malenka RC, Nicoll RA. Development of excitatory circuitry in the hippocampus. J Neurophysiol 79: 2013-2024, 1998.

Hyson RL. The analysis of interaural time differences in the chick brain stem. Physiol Behav 86: 297-305, 2005.

Isaac JT, Crair MC, Nicoll RA, Malenka RC. Silent synapses during development of thalamocortical inputs. Neuron 18: 269-280, 1997.

Jackson H, Hackett JT, Rubel EW. Organization and development of brain stem auditory nuclei in the chick: ontogeny of postsynaptic responses. $J$ Comp Neurol 210: 80-86, 1982.

Joshi I, Wang LY. Developmental profiles of glutamate receptors and synaptic transmission at a single synapse in the mouse auditory brain stem. $J$ Physiol 540: 861-873, 2002.

Kasanetz F, Manzoni OJ. Maturation of excitatory synaptic transmission of the rat nucleus accumbens from juvenile to adult. J Neurophysiol 101: 2516-2527, 2009.

Kosowski AR, Liljequist S. The NR2B-selective N-methyl-d-aspartate receptor antagonist Ro 25-6981 [(+/-)-( $\left.\mathrm{R}^{*}, \mathrm{~S}^{*}\right)$-alpha-(4-hydroxyphenyl)-betamethyl-4-(phenylmethyl)-1-pipe ridine propanol] potentiates the effect of nicotine on locomotor activity and dopamine release in the nucleus accumbens. J Pharmacol Exp Ther 311: 560-567, 2004.

Kuba H, Ishii TM, Ohmori H. Axonal site of spike initiation enhances auditory coincidence detection. Nature 444: 1069-1072, 2006.

Kuba H, Koyano K, Ohmori H. Development of membrane conductance improves coincidence detection in the nucleus laminaris of the chicken. $J$ Physiol 540: 529-542, 2002a.

Kuba H, Koyano K, Ohmori H. Synaptic depression improves coincidence detection in the nucleus laminaris in brain stem slices of the chick embryo. Eur J Neurosci 15: 984-990, 2002b.

Kuba H, Yamada R, Fukui I, Ohmori H. Tonotopic specialization of auditory coincidence detection in nucleus laminaris of the chick. $J$ Neurosci 25: $1924-1934,2005$

Kuba H, Yamada R, Ohmori H. Evaluation of the limiting acuity of coincidence detection in nucleus laminaris of the chicken. $J$ Physiol 552: 611-620, 2003.

Kumar SS, Bacci A, Kharazia V, Huguenard JR. A developmental switch of AMPA receptor subunits in neocortical pyramidal neurons. $J$ Neurosci 22: 3005-3015, 2002. 
Lawrence JJ, Trussell LO. Long-term specification of AMPA receptor properties after synapse formation. J Neurosci 20: 4864-4870, 2000.

Liao D, Hessler NA, Malinow R. Activation of postsynaptically silent synapses during pairing-induced LTP in CA1 region of hippocampal slice. Nature 375: 400-404, 1995.

Lichtman JW, Colman H. Synapse elimination and indelible memory. Neuron 25: 269-278, 2000

Lu HC, Gonzalez E, Crair MC. Barrel cortex critical period plasticity is independent of changes in NMDA receptor subunit composition. Neuron 32: 619-634, 2001.

Lu T, Trussell LO. Development and elimination of endbulb synapses in the chick cochlear nucleus. J Neurosci 27: 808-817, 2007.

Lynch DR, Shim SS, Seifert KM, Kurapathi S, Mutel V, Gallagher MJ, Guttmann RP. Pharmacological characterization of interactions of RO 25-6981 with the NR2B (epsilon2) subunit. Eur J Pharmacol 416: 185195, 2001.

Magee JC, Cook EP. Somatic EPSP amplitude is independent of synapse location in hippocampal pyramidal neurons. Nat Neurosci 3: 895-903, 2000.

Monsivais P, Yang L, Rubel EW. GABAergic inhibition in nucleus magnocellularis: implications for phase locking in the avian auditory brain stem. $J$ Neurosci 20: 2954-2963, 2000.

Otis TS, Raman IM, Trussell LO. AMPA receptors with high $\mathrm{Ca}^{2+}$ permeability mediate synaptic transmission in the avian auditory pathway. $J$ Physiol 482: 309-315, 1995.

Parks TN. The AMPA receptors of auditory neurons. Hear Res 147: 77-91, 2000.

Parks TN, Rubel EW. Organization and development of brain stem auditory nuclei of the chicken: organization of projections from $n$. magnocellularis to n. laminaris. J Comp Neurol 164: 435-448, 1975.

Parks TN, Rubel EW. Organization and development of the brain stem auditory nuclei of the chicken: primary afferent projections. J Comp Neurol 180: 439-448, 1978.

Pellegrini-Giampietro DE, Gorter JA, Bennett MV, Zukin RS. The GluR2 (GluR-B) hypothesis: $\mathrm{Ca}(2+)$-permeable AMPA receptors in neurological disorders. Trends Neurosci 20: 464-470, 1997.

Petralia RS, Esteban JA, Wang YX, Partridge JG, Zhao HM, Wenthold RJ, Malinow R. Selective acquisition of AMPA receptors over postnata development suggests a molecular basis for silent synapses. Nat Neurosci 2: 31-36, 1999.

Philpot BD, Sekhar AK, Shouval HZ, Bear MF. Visual experience and deprivation bidirectionally modify the composition and function of NMDA receptors in visual cortex. Neuron 29: 157-169, 2001.

Pliss L, Yang H, Xu-Friedman MA. Context-dependent effects of NMDA receptors on precise timing information at the endbulb of held in the cochlear nucleus. J Neurophysiol 102: 2627-2637, 2009.

Pruss RM, Akeson RL, Racke MM, Wilburn JL. Agonist-activated cobalt uptake identifies divalent cation-permeable kainate receptors on neurons and glial cells. Neuron 7: 509-518, 1991.

Quinlan EM, Olstein DH, Bear MF. Bidirectional, experience-dependent regulation of N-methyl-d-aspartate receptor subunit composition in the rat visual cortex during postnatal development. Proc Natl Acad Sci USA 96: 12876-12880, 1999.

Rajan I, Cline HT. Glutamate receptor activity is required for normal development of tectal cell dendrites in vivo. J Neurosci 18: 7836-7846, 1998.

Rajan I, Witte S, Cline HT. NMDA receptor activity stabilizes presynaptic retinotectal axons and postsynaptic optic tectal cell dendrites in vivo. $J$ Neurobiol 38: 357-368, 1999.

Raman IM, Trussell LO. The kinetics of the response to glutamate and kainate in neurons of the avian cochlear nucleus. Neuron 9: 173-186, 1992.

Raman IM, Zhang S, Trussell LO. Pathway-specific variants of AMPA receptors and their contribution to neuronal signaling. J Neurosci 14: $4998-5010,1994$
Reyes AD, Rubel EW, Spain WJ. In vitro analysis of optimal stimuli for phase-locking and time-delayed modulation of firing in avian nucleus laminaris neurons. J Neurosci 16: 993-1007, 1996.

Rumbaugh G, Vicini S. Distinct synaptic and extrasynaptic NMDA receptors in developing cerebellar granule neurons. J Neurosci 19: 10603-10610, 1999.

Rumpel S, Kattenstroth G, Gottmann K. Silent synapses in the immature visual cortex: layer-specific developmental regulation. J Neurophysiol 91: 1097-1101, 2004.

Ruthazer ES, Cline HT. Insights into activity-dependent map formation from the retinotectal system: a middle-of-the-brain perspective. J Neurobiol 59: 134-146, 2004.

Sabatini BL, Regehr WG. Timing of synaptic transmission. Annu Rev Physiol 61: 521-542, 1999.

Sanchez JT, Gans D, Wenstrup JJ. Contribution of NMDA and AMPA receptors to temporal patterning of auditory responses in the inferior colliculus. J Neurosci 27: 1954-1963, 2007.

Sin WC, Haas K, Ruthazer ES, Cline HT. Dendrite growth increased by visual activity requires NMDA receptor and Rho GTPases. Nature 419: 475-480, 2002.

Slee SJ, Higgs MH, Fairhall AL, Spain WJ. Tonotopic tuning in a sound localization circuit. J Neurophysiol 103: 2857-2875.

Smith DJ, Rubel EW. Organization and development of brain stem auditory nuclei of the chicken: dendritic gradients in nucleus laminaris. J Comp Neurol 186: 213-239, 1979.

Smith ZD. Organization and development of brain stem auditory nuclei of the chicken: dendritic development in N. laminaris. J Comp Neurol 203: 309-333, 1981.

Tang YZ, Carr CE. Development of NMDA R1 expression in chicken auditory brain stem. Hear Res 191: 79-89, 2004.

Tang YZ, Carr CE. Development of $N$-methyl-d-aspartate receptor subunits in avian auditory brain stem. J Comp Neurol 502: 400-413, 2007.

Tovar KR, Westbrook GL. The incorporation of NMDA receptors with a distinct subunit composition at nascent hippocampal synapses in vitro. $J$ Neurosci 19: 4180-4188, 1999.

Trussell L. Control of time course of glutamatergic synaptic currents. Prog Brain Res 116: 59-69, 1998.

Trussell LO. Cellular mechanisms for preservation of timing in central auditory pathways. Curr Opin Neurobiol 7: 487-492, 1997.

Trussell LO. Synaptic mechanisms for coding timing in auditory neurons. Annu Rev Physiol 61: 477-496, 1999.

Turrigiano GG. The self-tuning neuron: synaptic scaling of excitatory synapses. Cell 135: 422-435, 2008.

Tzingounis AV, Wadiche JI. Glutamate transporters: confining runaway excitation by shaping synaptic transmission. Nat Rev Neurosci 8: 935-947, 2007.

Wang Y, Rubel EW. Rapid regulation of microtubule-associated protein 2 in dendrites of nucleus laminaris of the chick following deprivation of afferent activity. Neuroscience 154: 381-389, 2008.

Williams K. Interactions of polyamines with ion channels. Biochem J 325: 289-297, 1997.

Williams SR, Mitchell SJ. Direct measurement of somatic voltage clamp errors in central neurons. Nat Neurosci 11: 790-798, 2008.

Wu GY, Zou DJ, Rajan I, Cline H. Dendritic dynamics in vivo change during neuronal maturation. J Neurosci 19: 4472-4483, 1999.

Young SR, Rubel EW. Frequency-specific projections of individual neurons in chick brain stem auditory nuclei. J Neurosci 3: 1373-1378, 1983.

Zhou N, Parks TN. Pharmacology of excitatory amino acid neurotransmission in nucleus laminaris of the chick. Hear Res 52: 195-200, 1991.

Zhou N, Taylor DA, Parks TN. Cobalt-permeable non-NMDA receptors in developing chick brain stem auditory nuclei. Neuroreport 6: 2273-2276, 1995. 